Article

\title{
Variations of Macro- and Microelements in Yellow-Fleshed Cassava (Manihot esculenta Crantz) Genotypes as a Function of Storage Root Portion, Harvesting Time, and Sampling Method
}

\author{
Emmanuel Oladeji Alamu ${ }^{1, *}{ }^{\oplus}$, Busie Maziya-Dixon ${ }^{2}$, Consent Sibeso ${ }^{1}$, Elizabeth Parkes ${ }^{1}$ and \\ Alfred Gilbert Dixon ${ }^{1}$ (D) \\ 1 International Institute of Tropical Agriculture, Southern Africa, Research and Administration Hub (SARAH) \\ Campus, P.O. Box 310142, Chelstone, Lusaka 10101, Zambia; c.sibeso@cgiar.org (C.S.); \\ e.parkes@cgiar.org (E.P.); a.dixon@cgiar.org (A.G.D.) \\ 2 International Institute of Tropical Agriculture, PMB 5230, Ibadan 20001, Oyo State, Nigeria; \\ b.maziya-dixon@cgiar.org \\ * Correspondence: oalamu@cgiar.org; Tel.: +260-976-338-710; Fax: +260-44-208-711-3786
}

Received: 18 June 2020; Accepted: 7 July 2020; Published: 5 August 2020

Featured Application: This study has provided information on the quantitative and qualitative information on the distribution of macro and trace elements and their concentrations within the cassava tuber (proximal, middle, and distal root portions). The data on the minerals of yellow-fleshed cassava roots could contribute to food composition databases. The knowledge generated would be of interest to researchers (cassava breeders and food scientists), food processors, dieticians, and policymakers in programming interventions. Yellow-fleshed cassava could be an alternative source of macro- and microelements for both humans and livestock.

\begin{abstract}
The correct estimation of the mineral content of cassava (Manihot esculenta) genotypes is vital from a nutritional point of view. This study evaluated the effects of the storage root section, maturity, and sampling method on the macro- and microelements in yellow-fleshed cassava root genotypes. In total, 44 genotypes were grown in replicated field trials of 2 sets (set 25 and set 19) and were harvested at 9 and 12 months after planting. Two sampling methods, sampling with a cork borer $(\mathrm{A}=$ proximal, $\mathrm{B}=$ middle, $\mathrm{C}=$ distal or method 1$)$ and sampling without a cork borer $(\mathrm{L}=$ Longitudinal or method 2$)$, were used. The minerals of the samples from the two methods were determined using inductively coupled optical emission spectrometry (ICP-OES). K and $\mathrm{Mn}$ were the most abundant minerals, and $\mathrm{Na}, \mathrm{Mo}$, and $\mathrm{Co}$ were the least abundant. Genotype, method, and maturity had a strong influence on mineral concentrations. Harvesting time affected the concentration level of some macro- and microelements in cassava roots. Additionally, $\mathrm{Ca}, \mathrm{Mg}, \mathrm{K}, \mathrm{P}$, $\mathrm{Mn}, \mathrm{Cu}, \mathrm{Ni}$, and $\mathrm{Zn}$ contents were significantly $(p<0.05)$ higher in the proximal and middle portions for method 1. K and P and Mn and B were positively correlated, but K and Na and Fe and Ni were negatively associated.
\end{abstract}

Keywords: macroelements; microelements; yellow-fleshed cassava; genotypes; storage portions; sampling method; maturity

\section{Introduction}

Minerals are the constituents that remain as ash after the combustion of plant and animal tissues. Minerals can be classified as primary (macro) elements, trace elements, and ultra-trace elements, and the 
main elements are essential for human beings in amounts $>50 \mathrm{mg} /$ day. Essential macroelements, such as $\mathrm{Na}, \mathrm{K}, \mathrm{Mg}, \mathrm{P}$, and $\mathrm{S}$, are found in cassava and can play a vital role in the human body. Trace elements are naturally occurring inorganic substances required in humans in amounts $<100 \mathrm{mg} /$ day. They are essential components of biological structures and have an essential effect on and play a vital role in a variety of the processes necessary for life by mediating vital biochemical reactions [1].

Still, the potential of cassava as a significant source of these elements is yet to be fully exploited. Cassava (Manihot esculenta Crantz) is a tuberous root that belongs to the Euphorbiaceae family. It is also known as manioc, tapioca, or yucca. Cassava is adaptable to a variety of environmental conditions and is an essential source of nutrients and energy. It is the third-largest source of carbohydrates after rice and maize [2,3]. Although cassava leaves are consumed at times for food and medicinal purposes, its tuber (the swollen root of the plant) is the standard part of the plant that is consumed widely [4]. It is argued that more than two-thirds of the total production of cassava is used as food for humans, with lesser amounts being used for animal feed and industrial purposes [5]. Cassava can be consumed either in its natural form (i.e., boiled) or in a variety of industrially or traditionally processed forms, known under various names depending on the preferences and local customs. Cassava flour is one of these products, as well as chips and starch [6]. The root flour (high-quality cassava flour, HQCF) is gaining recognition as a suitable wheat flour substitute in biscuits and the fast-food industry. It is an essential raw material in the food industry preparation of a wide array of value-added products, such as bread, cookies, confectionery, pasta, and couscous-like products, and the production of adhesives. It is also used as a thickener in foods that are not subject to rigorous processing conditions. Indeed, although cassava flour has a low nutritional value, it remains an essential food in several parts of the world, as it is less expensive than wheat and can be used to produce a variety of food products [7-9].

Both macro and trace minerals are needed for the growth and maintenance of human body structures. The human body uses minerals for the proper composition of bone and blood, as well as the maintenance of normal cell function. Thus, diets rich in minerals are essential for proper growth and development. Further, as reported, essential macroelements, such as $\mathrm{Ca}, \mathrm{K}, \mathrm{Mg}$, and $\mathrm{P}$, which are found in cassava roots, play a vital role in the human body [10]. Ca, for example, is responsible for several metabolic functions, such as blood coagulation, muscle contraction regulation, hormone secretion, and neurotransmission. $\mathrm{Mg}$ is a macroelement that participates in energy metabolism and is involved in protein synthesis. Daily intake of sodium averages from $2.5 \mathrm{~g}$ (females) to $3.3 \mathrm{~g}$ (males); the adult average requirement ranges from 1.3 to $1.6 \mathrm{~g} /$ day (equal to $3.3-4.0 \mathrm{~g} / \mathrm{day} \mathrm{NaCl}$ ). The intake of too little or too much sodium can result in severe disorders. From a nutritional standpoint, only the excessive consumption of sodium is of importance because it can lead to hypertension. A low intake of sodium can be achieved by a non-salty diet or by using diet salt [11].

Likewise, trace elements also have several important roles in human bodies. Some are essential for enzyme reactions, where they attract and facilitate the conversion of substrate molecules to specific end-products. Moreover, some of them donate or accept electrons in redox reactions that are of primary importance in the generation and utilization of metabolic energy. Some of them have structural roles and responsibilities for the stability of critical biological molecules. Furthermore, some trace elements have necessary actions throughout biological processes, for example, iron (Fe), which can bind, transport, and release oxygen in the body. The established recommended daily amount (RDA) for Fe is $13.7-15.1 \mathrm{mg} /$ day in children, $19.3-20.5 \mathrm{mg}$ /day in men, and $17.0-18.9 \mathrm{mg} /$ day in women, and for $\mathrm{Zn}$, it is $8 \mathrm{mg} /$ day for women and $11 \mathrm{mg} /$ day for men. It is particularly vital for healthy skin and is essential for a healthy immune system and resistance to infection. Zn plays a crucial role in growth and cell division, where it is required for protein and DNA synthesis, in insulin activity, in the metabolism of the ovaries and testes, and liver function. Mn helps the body to form connective tissue, bones, blood-clotting factors, and sex hormones. It also plays a role in fat and carbohydrate metabolism, calcium absorption, and blood sugar regulation. Mn is also necessary for a healthy brain and nerve function. Co is an essential trace element for the human body, where it is a crucial constituent of 
cobalamin (the scientific name of vitamin B12). It also has a substantial role in the formation of amino acids and neurotransmitters [1].

It has been reported in the literature that the mineral element contents of plants are affected by the plant cultivar, soil conditions, weather conditions during the growing season, use of fertilisers, and the stage of plant maturity at harvest [12]. However, the variability of values of macroelements reported in the literature for cassava roots has revealed discrepancies that surpass those expected from the effects of the factors above but could be due to analytic inaccuracy, especially from the sampling method used. The correct estimation of the macro- and microelement content of cassava genotypes is vital from a nutritional point of view, especially for controlling and improving mineral intake in sub-Saharan Africa. There is a scarcity of information on the spatial distribution of both macro- and microelement contents of yellow-fleshed cassava storage roots. Based on this background, the present study aimed to evaluate the spatial distribution of macro and microelements in yellow-fleshed cassava root genotypes and establish the effect of the sampling method and harvesting time on their content. The knowledge generated from this study can be used by researchers, processors, dieticians, and policymakers in programming interventions.

\section{Materials and Methods}

\subsection{Collection of Genetic Materials}

A total of 39 yellow cassava genotypes from unlimited yield trials (UYTs) with $\beta$ carotene-rich storage roots (yellow-fleshed) and five white-fleshed varieties (as checks) were grown in replicated field trials at the research farm of the International Institute of Tropical Agriculture (IITA), Ibadan, Nigeria. The 44 genotypes and varieties were planted in two sets. The set 1 trial comprised 22 genotypes with $\beta$-carotene-enriched roots and three check genotypes with white roots (30,572, TME 1, and 91/02324). The set 2 trial comprised 17 genotypes with $\beta$-carotene-enriched roots and two check varieties with white roots (30,572 and 91/02324). Through the crossbreeding of the wild-type varieties, the cassava breeders have been trying to improve the nutritional value, especially the $\beta$-carotene-enriched roots. The two sets were different from each other by the source (parent lines with different quality traits) used for the breeding crosses, which could dictate the mineral chemical properties of the genotypes. The experiments were planted during the rainy season in July and grown under rainfed conditions without the application of fertilisers or herbicides. The storage roots were harvested at 9 and 12 months after planting (MAP). Only the two middle rows were harvested per plot, and the cassava roots processed were collected from only one replication. Three plants per genotype were harvested, and six cassava roots of different sizes (large, medium, and big) were randomly selected per genotype and placed in a labelled polyethene bag and transferred to the laboratory for sample preparation for analysis.

\subsection{Preparation of Samples}

\subsubsection{Sampling Method 1}

The method reported by Maziya-Dixon et al. [13] was used for the first sampling method. In the laboratory, three storage roots of different sizes (large (900-2300 g), medium (500 to $899 \mathrm{~g}$ ), and small (200 to 499 g)) were selected, washed thoroughly with potable water to remove dirt and sand particles, and air-dried on a clean concrete floor for about 5-10 $\mathrm{min}$ at atmospheric conditions. The storage roots were peeled manually using a stainless-steel knife and rinsed with deionized water. A cork borer (size 8) was used to bore through the storage roots, with the depth ranging from 4.6 to $9.7 \mathrm{~cm}$, 3.8 to $7.1 \mathrm{~cm}$, and 3.6 to $6.4 \mathrm{~cm}$ for the proximal, middle, and distal parts of the roots, respectively, and each portion was homogenized [13]. The rinsing of tubers and the sampling method guaranteed the elimination of mineral contaminants from the dirt and sand adhered to the tubers. 


\subsubsection{Sampling Method 2}

The second sampling method was also described by Maziya-Dixon et al. [13]. A new set of the 3 storage roots of different sizes (large, medium, and small) were selected, washed thoroughly with potable water to remove dirt and adhered sand particles, and air-dried on a clean concrete floor. The storage roots were peeled manually using a stainless-steel knife, rinsed with deionized water, and cut longitudinally (from the proximal end to the distal end) into four equal parts. Two opposite sections from each root of each genotype were taken, combined, manually chopped into small pieces, and mixed thoroughly.

\subsection{Determination of Macro- and Microelement Content}

The samples for the determination of macro- and microelements were taken from the batch samples from the above-described two sampling methods. The samples were rinsed with deionized water, and placed in a petri dish and dried in an uncorroded conventional oven at $40^{\circ} \mathrm{C}$ for 3 days. After drying, the samples were packed in labelled mineral-free paper envelopes. The macro- and microelement contents were analysed using inductively coupled optical emission spectrometry (ICP-OES) according to the validated method described by Wheal et al. [14]. A radial view Spectro Ciros CCD ICP-OES (Spectro Analytical Instruments, Kleve, Germany) was used, and all samples were digested with the closed-tube nitric acid/hydrogen peroxide digestion method as described elsewhere by Wheal et al. [14]. About $0.30 \mathrm{~g}$ of the dried sample was weighed to $1 \mathrm{mg}$ into $50-\mathrm{mL}$ screw-cap polypropylene tubes, and $2.0 \mathrm{~mL}$ of $\mathrm{HNO}_{3}$ and $0.5 \mathrm{~mL}$ of $\mathrm{H}_{2} \mathrm{O}_{2}$ were added to initiate the sample digestion. The digestion was done at $125^{\circ} \mathrm{C}$ for $120 \mathrm{~min}$ using 72-position DigiPrep digestion blocks (SCP Scientific, Baie D'Urf e, Quebec, Canada). The samples were made to a final volume of $25 \mathrm{~mL}$ with $18 \mathrm{M} \Omega . \mathrm{cm}$ water. The sample flow rate was $2.0 \mathrm{~mL} / \mathrm{min}$, and the total analysis time per sample was approximately $2.5 \mathrm{~min}$. The calibration curves for all elements were created from mixtures of high-purity single-element standard solutions in a $4 \%(v / v) \mathrm{HNO}_{3}$ matrix. Background correction was applied to all wavelengths, and spectral interferences were applied where required using software algorithms [14]. A drift correction solution (a mixture of all elements prepared in $4 \% \mathrm{HNO}_{3}$ ) was analysed every 25 samples to account for within-run variation in the flows, etc. Additionally, the blank subtraction, the drift correction, and other data processing (mass and volume adjustments) were performed offline [14]. Six different reference materials (RMs) were obtained from the US National Institute of Standards and Technology (NIST, Gaithersburg, MD, USA) and used to establish the quality of the data [14].

Each sample was run in duplicate. The macroelements identified in all the genotypes and varieties investigated were calcium $(\mathrm{Ca})$, magnesium $(\mathrm{Mg})$, sodium $(\mathrm{Na})$, potassium $(\mathrm{K})$, phosphorus $(\mathrm{P})$, and sulphur (S). The microelements identified in all the genotypes and varieties investigated were iron $(\mathrm{Fe})$, manganese $(\mathrm{Mn})$, boron $(\mathrm{B})$, copper $(\mathrm{Cu})$, molybdenum $(\mathrm{Mo})$, cobalt $(\mathrm{Co})$, nickel $(\mathrm{Ni})$, zinc $(\mathrm{Zn})$, and aluminium (Al).

\subsection{Statistical Analysis}

Data were subjected to analysis of variance, descriptive statistics, and Pearson correlation analysis using the Statistical Analysis System software (SAS) [15]. Means were separated using Fisher's protected least significance difference test at $p<0.05$.

\section{Results and Discussion}

3.1. Macro- and Micromineral Contents of Yellow-Fleshed Cassava Roots Across Genotypes, Locations, and Sets 1 and 2

The macro- and micromineral contents of yellow-fleshed cassava root across all the genotypes and locations are presented in Table 1 . The most abundant mineral in cassava storage roots was potassium (K), with concentrations ranging from 4300 to $26,000 \mathrm{mg} / \mathrm{kg}$ with an overall mean of 
$12,116 \pm 3543.58 \mathrm{mg} / \mathrm{kg}$ for trial 1 , and ranged from 6300 to $38,000 \mathrm{mg} / \mathrm{kg}$ with a mean value of $17,883.60 \pm 4898.77 \mathrm{mg} / \mathrm{kg}$ for trial 2 . However, the least abundant mineral was sodium $(\mathrm{Na})$, with concentrations ranging from 15.68 to $2100 \mathrm{mg} / \mathrm{kg}$ with an overall mean of $228.22 \pm 316.74 \mathrm{mg} / \mathrm{kg}$ for trial 1, and from 11.47 to $186.73 \mathrm{mg} / \mathrm{kg}$ with a total mean value of $39.19 \pm 23.41 \mathrm{mg} / \mathrm{kg}$ for trial 2. The results also showed that trial 2 had higher concentrations of calcium (Ca), magnesium $(\mathrm{Mg})$, and potassium (K) than trial 1. Still, trial 1 sets had a higher content of $\mathrm{Na}, \mathrm{P}$ (phosphorous), and $\mathrm{S}$ (sulphur). It could be deduced that the storage roots of cassava could be a good source of these macroelements, especially potassium. Diets rich in minerals are essential for proper growth and development [11]. This result agrees with Aro et al. [16], who reported that the highest mineral content of the cassava samples studied was K $(269 \mathrm{mg} / \mathrm{kg})$, but contradicts with Bamidele et al. [17]. Among the mineral compositions of the four cassava samples they studied, $\mathrm{Mg}$ was the highest mineral found in all four cassava samples investigated, with values ranging between 185.1 and $321.5 \mathrm{mg} / \mathrm{kg}$. Manano et al. [18] studied some local and improved high-yielding and cassava mosaic disease (CMD)-resistant cassava varieties grown in Uganda. The findings of this study indicated that the levels of minerals differed with the cassava variety. The cassava yellow root genotypes for both trials and 1 and 2 had very low $\mathrm{Na}$, and this indicates that these genotypes could form part of a low-sodium diet. However, $\mathrm{K}$ regulates the osmotic pressure within the cell, and is involved in cell membrane transport and the activation of several glycolytic and respiratory enzymes. Potassium intake in a regular diet is $2-5.9 \mathrm{~g} /$ day. The minimum daily requirement is estimated to be $782 \mathrm{mg}$. It implies that cassava, with a high level of $\mathrm{K}$ and low $\mathrm{Na}$, might be an ideal crop to consume to reduce hypertension, although this needs further research evidence.

The most abundant mineral in cassava storage roots was manganese (Mn), with concentrations ranging from 7.390 to $39.990 \mathrm{mg} / \mathrm{kg}$ with an overall mean of $16.511 \pm 6.200 \mathrm{mg} / \mathrm{kg}$ for trial 1, and ranging from 4.540 to $42.680 \mathrm{mg} / \mathrm{kg}$ with a mean value of $13.383 \pm 4.698 \mathrm{mg} / \mathrm{kg}$ for trial 2. However, the least abundant minerals were molybdenum $(\mathrm{Mo})$ and cobalt $(\mathrm{Co})$, with concentrations ranging from 0.600 to $0.700 \mathrm{mg} / \mathrm{kg}$ with an overall mean of $0.694 \pm 0.025$ and $0.675 \pm 0.043 \mathrm{mg} / \mathrm{kg}$ for trial 1, and ranging from 0.600 to $3.000 \mathrm{mg} / \mathrm{kg}$ with total mean values of $0.708 \pm 0.237$ and $0.696 \pm 0.239 \mathrm{mg} / \mathrm{kg}$ for trial 2, respectively. The results also showed that all the minerals in trial 2 had higher concentrations than trial 1 , as found for macrominerals. It could be deduced that the storage roots of cassava could be a good source of these microelements, especially Mn.

\subsection{Mean Squares (MS) of Macro- and Microelements of Yellow-Fleshed Cassava Root}

The effects of genotypes, maturity, and method of sampling on the macro- and microelements of yellow-fleshed cassava roots are presented in Tables 2 and 3. For trial 1, the ANOVA showed that genotype, method, and genotype $\times$ maturity interaction had a strong significant $(p<0.001)$ effect on all the macrominerals studied. However, maturity had no significant effect $(p>0.05)$ on $\mathrm{Mg}, \mathrm{Na}$, $\mathrm{K}$, and S. Genotype $\times$ maturity interaction had a significant effect $(p<0.001)$ on all the minerals except for Ca. Only Mg, S, and Na showed a significant difference $(p<0.001)$ for genotype $\times$ method interaction. For maturity $\times$ method interaction, only $\mathrm{Ca}$ and Na showed a significant difference at $(p<0.001)$. For the data set 2 trial, there was a highly significant effect $(p<0.001)$ of genotype and genotype $\times$ maturity interaction on all the macroelements. Maturity and method had no significant effect $(p<0.05)$ on $\mathrm{Mg}$ and $\mathrm{Ca}$, respectively. Though, genotype $\times$ method interaction had a significant effect (at $p<0.001)$ only on $\mathrm{Na}$; maturity $\times$ method interaction showed no significant effect $(p<0.05)$ across all the minerals investigated. Thus, for both trials 1 and 2, the independent variables (genotype and maturity) explained the variations observed for all the dependent variables ( $\mathrm{Ca}, \mathrm{Mg}, \mathrm{Na}, \mathrm{K}, \mathrm{P}$, and S) rather than the basic mean. 
Table 1. Summary of mineral (macro and micro) contents of yellow cassava root genotypes from trial sets 1 and 2.

\begin{tabular}{|c|c|c|c|c|c|c|c|c|c|c|c|c|c|c|c|}
\hline \multirow{2}{*}{ Statistic } & $\mathrm{Ca}$ & $\mathbf{M g}$ & $\mathrm{Na}$ & $\mathbf{K}$ & $\mathbf{P}$ & Su & $\mathrm{Fe}$ & Mn & B & $\mathrm{Cu}$ & Mo & Co & $\mathbf{N i}$ & Zn & Al \\
\hline & \multicolumn{15}{|c|}{ Parameters (mg/kg) } \\
\hline \multicolumn{16}{|l|}{ Trial Set 1} \\
\hline Minimum & 440.0 & 370.0 & 15.7 & 4300.0 & 600.0 & 123.7 & 3.69 & 7.39 & 2 & 0.85 & 0.6 & 0.6 & 0.9 & 4.23 & 4 \\
\hline Maximum & 2700.0 & 3000.0 & 2100.0 & $26,000.0$ & 3300.0 & 530.0 & 21.91 & 39.99 & 3.8 & 8.49 & 0.7 & 0.7 & 6.08 & 17.31 & 9.6 \\
\hline Mean & 1149.7 & 1184.5 & 228.2 & $12,116.0$ & 1233.0 & 290.9 & 8.46 & 16.51 & 2.16 & 3.49 & 0.69 & 0.68 & 2.3 & 8.69 & 5.04 \\
\hline Standard deviation $(n-1)$ & 455.0 & 405.0 & 316.8 & 3543.6 & 471.3 & 67.3 & 3.35 & 6.2 & 0.33 & 1.39 & 0.03 & 0.04 & 0.79 & 2.23 & 0.68 \\
\hline Standard error of the mean & 28.8 & 25.6 & 20.0 & 224.1 & 29.8 & 4.3 & 0.24 & 0.44 & 0.02 & 0.1 & 0 & 0 & 0.06 & 0.16 & 0.05 \\
\hline Lower bound on mean (95\%) & 1093.0 & 1134.0 & 188.8 & $11,674.6$ & 1174.4 & 282.5 & 7.99 & 15.65 & 2.12 & 3.3 & 0.69 & 0.67 & 2.19 & 8.38 & 4.94 \\
\hline Upper bound on mean (95\%) & 1206.3 & 1234.9 & 267.7 & $12,557.4$ & 1291.8 & 299.3 & 8.92 & 17.38 & 2.21 & 3.68 & 0.7 & 0.68 & 2.41 & 9 & 5.13 \\
\hline \multicolumn{16}{|l|}{ Trial Set 2} \\
\hline Minimum & 530.0 & 420.0 & 11.5 & 6300.0 & 540.0 & 146.5 & 4.1 & 4.54 & 2 & 1.78 & 0.6 & 0.6 & 0.9 & 4.52 & 4 \\
\hline Maximum & 5200.0 & 2900.0 & 186.7 & $38,000.0$ & 2600.0 & 440.0 & 41.42 & 42.68 & 9.82 & 26.52 & 3 & 3 & 6.48 & 20.52 & 24.57 \\
\hline Mean & 1444.7 & 1129.7 & 39.2 & $17,883.6$ & 1112.9 & 271.1 & 9.52 & 13.38 & 2.52 & 4.8 & 0.71 & 0.7 & 1.96 & 9.66 & 5.29 \\
\hline Standard deviation $(n-1)$ & 622.5 & 371.6 & 23.4 & 4898.8 & 344.5 & 65.3 & 4.48 & 4.7 & 0.87 & 2.38 & 0.24 & 0.24 & 0.84 & 3.27 & 2.73 \\
\hline Standard error of the mean & 45.3 & 27.0 & 1.7 & 356.3 & 25.1 & 4.7 & 0.36 & 0.38 & 0.07 & 0.19 & 0.02 & 0.02 & 0.07 & 0.27 & 0.22 \\
\hline Lower bound on mean (95\%) & 1355.4 & 1076.4 & 35.8 & $17,180.7$ & 1063.4 & 261.8 & 8.8 & 12.63 & 2.38 & 4.41 & 0.67 & 0.66 & 1.82 & 9.13 & 4.85 \\
\hline Upper bound on mean (95\%) & 1534.0 & 1183.0 & 42.6 & $18,586.5$ & 1162.3 & 280.5 & 10.24 & 14.14 & 2.66 & 5.18 & 0.75 & 0.73 & 2.09 & 10.19 & 5.73 \\
\hline
\end{tabular}


Table 2. Mean squares (MS) of macroelements of yellow-fleshed cassava root.

\begin{tabular}{|c|c|c|c|c|c|c|c|}
\hline \multirow{2}{*}{ Source } & DF & $\mathrm{Ca}$ & $\mathrm{Mg}$ & $\mathrm{Na}$ & $\mathbf{K}$ & $\mathbf{P}$ & $S$ \\
\hline & \multicolumn{7}{|c|}{ Parameters (mg/kg) } \\
\hline Trail Set 1 & & & & & & & \\
\hline Genotype & 24 & $1,094,636.9^{* * *}$ & $870,988^{* * * *}$ & $433,588.7^{* * *}$ & $67,299,657.4^{* * *}$ & $948,782.6^{* * *}$ & $31,643.2^{* * *}$ \\
\hline Maturity & 1 & $6,634,645.5 * * *$ & $54,562.8$ & 245.4 & $1,474,560$ & $4,725,187.6^{* * *}$ & 1413.6 \\
\hline Method & 4 & $645,224.4^{* * *}$ & $1,260,184.4^{* * * *}$ & $627,252.2^{* * *}$ & $14,453,8100^{* * *}$ & $90,8662.4^{* * *}$ & $10,790.2 * * *$ \\
\hline Genotype $\times$ Maturity & 24 & $223,944.8$ & $275,492.7^{* * * *}$ & $139,095.4^{* * *}$ & $14,043,235.9^{* * *}$ & $545,051.5^{* * *}$ & $4920^{* * *}$ \\
\hline Genotype $\times$ Method & 96 & $58,200.3$ & $52,372.4 * *$ & $52,509.3^{* *}$ & $3,109,278.3$ & $53,274.7$ & 1280.4 * \\
\hline Maturity $\times$ Method & 4 & $184,934.5^{* * *}$ & 8869.2 & $109,676.5^{* *}$ & $6,790,460$ & $11,3824.6$ & 521.2 \\
\hline Error & 96 & $45,495.2$ & $33,198.6$ & $33,832.3$ & $2,803,823.4$ & $57,485.7$ & 846.7 \\
\hline \multicolumn{8}{|l|}{ Trial Set 2} \\
\hline Genotype & 18 & $2,069,831.8^{* * *}$ & $766,222.2^{* * *}$ & $2390.6^{* * *}$ & $6,9316,383.5^{* * *}$ & $418,518.3^{* * *}$ & $22,729.8^{* * *}$ \\
\hline Maturity & 1 & $8,586,896^{* * *}$ & 8351.6 & $1233.1 *$ & $30,2441,860.2^{* * *}$ & $3,941,892.3^{* * *}$ & $86,920.9 * * *$ \\
\hline Method & 4 & $2059,09.1$ & $432,610.7^{* * *}$ & $1075.3^{* * *}$ & $223,459,232.4^{* * *}$ & $566,446.7^{* * *}$ & $18,305.5^{* * *}$ \\
\hline Genotype $\times$ Maturity & 18 & $540,855.2 * * *$ & $223,119.8^{* * *}$ & $508.9 * *$ & $41,997,484.4^{* * *}$ & $230,692.9^{* * *}$ & $3301.1^{* * *}$ \\
\hline Genotype $\times$ Method & 72 & $117,288.9$ & $47,556.3$ & 395.1 ** & $9,120,322.1$ & $32,886.3$ & 1144.7 \\
\hline Maturity $\times$ Method & 4 & $108,416.7$ & 8194.8 & 61.6 & $16,541,188.4$ & $14,550.8$ & 1102 \\
\hline Error & 72 & $105,165.3$ & 40982.7 & 230.6 & $8,178,619.1$ & $27,741.9$ & 1255.8 \\
\hline
\end{tabular}

ns, not significant at $p>0.05 ;{ }^{*}$, significant $p<0.05 ;{ }^{* *}$, significant at $p<0.01 ;{ }^{* * *}$, significant at $p<0.001$.

Table 3. Mean squares (MS) of microelements of yellow-fleshed cassava root.

\begin{tabular}{|c|c|c|c|c|c|c|c|c|c|c|}
\hline \multirow{2}{*}{ Source } & DF & $\mathrm{Fe}$ & Mn & B & $\mathrm{Cu}$ & Mo & Co & $\mathrm{Ni}$ & $\mathrm{Zn}$ & Al \\
\hline & \multicolumn{10}{|c|}{ Parameters (mg/kg) } \\
\hline \multicolumn{11}{|l|}{ Trial Set 1} \\
\hline Clone_Name & 24 & $22.869^{* * *}$ & $146.01^{* * *}$ & $0.216^{* * *}$ & $7.538^{* * *}$ & $0.001 * * *$ & 0.002 & $1.919 * * *$ & $13.665^{* * *}$ & 0.782 \\
\hline Method & 3 & $65.053^{* * *}$ & $64.268 *$ & 0.127 & $18.985^{* * *}$ & 0 & 0.001 & $2.47^{* * *}$ & $51.981 * * *$ & 1.05 \\
\hline Clone_Name $\times$ Maturity & 24 & $12.51 * * *$ & $51.154 * *$ & $0.151^{* * *}$ & $2.062 * * *$ & $0.002 * * *$ & $0.003 * *$ & $1.399 * * *$ & $7.029 * * *$ & 0.419 \\
\hline Clone_Name $\times$ Method & 72 & 4.075 & 13.67 & 0.048 & 0.596 & 0 & 0.002 & $0.221 *$ & 1.543 & 0.336 \\
\hline \multicolumn{11}{|l|}{ Trial Set 2} \\
\hline Clone_Name & 18 & $54.608^{* * *}$ & $89.043^{* * *}$ & $1.348^{* * *}$ & $13.281^{* * *}$ & 0.044 & 0.048 & $2.417^{* * *}$ & $41.364^{* * *}$ & $12.546^{* * *}$ \\
\hline Maturity & 1 & $123.164^{* * *}$ & $78.197 * * *$ & $18.365^{* * *}$ & 0.869 & 0.036 & 0.026 & $2.717 * * *$ & $214.769 * * *$ & $33.793 * *$ \\
\hline Method & 3 & 14.911 & $37.637 * * *$ & 0.208 & $28.696^{* * *}$ & 0.06 & 0.072 & $2.874^{* * *}$ & $65.65^{* * *}$ & 5.867 \\
\hline Clone_Name $\times$ Maturity & 18 & $34.644 * * *$ & $35.097 * * *$ & $1.009^{* *}$ & 5.653 & 0.081 & 0.085 & $0.697^{* * *}$ & $7.536^{* * *}$ & $16.783 * * *$ \\
\hline Clone_Name $\times$ Method & 54 & 12.511 & 8.671 & 0.504 & 4.184 & 0.053 & 0.051 & 0.361 & 2.703 & 4.786 \\
\hline Maturity × Method & 3 & 8.457 & 9.294 & 0.495 & 4.014 & $0.145 *$ & 0.134 & $0.98^{*}$ & $9.108 *$ & 8.092 \\
\hline
\end{tabular}

ns, not significant at $p>0.05 ;{ }^{*}$, significant $p<0.05 ;{ }^{* *}$, significant at $p<0.01 ;{ }^{* * *}$, significant at $p<0.001$.

Table 4 showed the effects of genotype, maturity, and method of sampling on the microelements of yellow-fleshed cassava roots. For trial 1, the ANOVA showed that genotype, maturity, method, genotype $\times$ maturity interaction, genotype $\times$ method interaction, and maturity $\times$ method interaction had a strong significant effect $(p<0.001)$ on all the minerals investigated except Al. However, genotype $\times$ method interaction had no significant effect $(p>0.05)$ on all the minerals except for Ni. From the dataset 2 trial, the ANOVA showed that genotype, maturity, method, genotype $\times$ maturity interaction, genotype $\times$ method interaction, and maturity $\times$ method interaction had a strong significant effect $(p<0.001)$ on all the minerals investigated except Co. However, the genotype $\times$ method interaction had no significant effect $(p<0.05)$ on all the minerals investigated. The study also revealed that maturity $\times$ method interaction had a significant difference $(p<0.001)$ on $\mathrm{Mo}, \mathrm{Ni}$, and $\mathrm{Zn}$. Both maturity and genotype $\times$ maturity interaction had a significant difference at $(p<0.001)$ on $\mathrm{Fe}, \mathrm{Mg}, \mathrm{B}, \mathrm{Ni}, \mathrm{Zn}$, and Al. Thus, for both trials 1 and 2, the independent variables (genotype and maturity) explained the variations observed for all the dependent variables $(\mathrm{Ca}, \mathrm{Mg}, \mathrm{Na}, \mathrm{K}, \mathrm{P}$, and $\mathrm{S})$ than the basic mean.

\subsection{Macromineral Concentrations of Yellow-Fleshed Cassava Roots by Genotype}

The means of the macromineral concentrations of yellow cassava roots (trial set 1 ) by genotype are shown in Table 4. For trial 1 , significant differences $(p<0.05)$ existed amongst the means of the macroelements for all the genotypes. However, genotypes 01/1224 $(2033 \mathrm{mg} / \mathrm{kg})$ and 01/1412 $(1742.3 \mathrm{mg} / \mathrm{kg})$ had the highest Ca concentrations among the samples investigated. The lowest Ca value $(596.3 \mathrm{mg} / \mathrm{kg})$ was found for TME1, the check variety. Genotypes 01/1368 $(676.2 \mathrm{mg} / \mathrm{kg})$ and $90 / 01554(650.5 \mathrm{mg} / \mathrm{kg})$ had the highest levels of $\mathrm{Na}$ in all the samples examined. However, the $\mathrm{Na}$ 
concentrations were found to be the lowest in genotype 94/0330 (44 mg/kg). For K, considerably higher concentrations were recorded in genotype 01/1662 (20160 mg/kg). However, the lowest K values were recorded in genotype $94 / 3030(8206.7 \mathrm{mg} / \mathrm{kg})$. The highest concentrations for $\mathrm{P}$ were recorded in genotype 01/1335 (1993.7 mg/kg). However, the P concentration was considerably lower in the 01/1371 $(813.3 \mathrm{mg} / \mathrm{kg}), 01 / 1412(807.7 \mathrm{mg} / \mathrm{kg}), 01 / 1413(847.7 \mathrm{mg} / \mathrm{kg})$, and 01/1442 (801 mg/kg) genotypes.

Table 4. Means of the macromineral concentrations of yellow cassava roots (trial Set 1 ) by genotype.

\begin{tabular}{|c|c|c|c|c|c|c|}
\hline \multirow{2}{*}{ Genotype } & $\mathrm{Ca}$ & $\mathrm{Mg}$ & $\mathrm{Na}$ & $\mathbf{K}$ & $\mathbf{P}$ & $\mathbf{S}$ \\
\hline & \multicolumn{6}{|c|}{ Parameters (mg/kg) } \\
\hline $01 / 1662$ & $1369.3 \mathrm{~cd}$ & 1081.7 defg & $61.0 \mathrm{fg}$ & $20,160.0 \mathrm{a}$ & $1791.7 \mathrm{ab}$ & 308.7 cdef \\
\hline $01 / 1115$ & 1090.0 defgh & $1441.3 \mathrm{bc}$ & 316.9 cdefg & $11,383.3$ defg & 1139.0 efgh & 310.7 cdef \\
\hline $01 / 1224$ & $2033.0 \mathrm{a}$ & $936.0 \mathrm{efgh}$ & $119.3 \mathrm{efg}$ & $11,050.0$ efgh & $989.7 \mathrm{gh}$ & $351.3 \mathrm{bc}$ \\
\hline $01 / 1235$ & 967.7 efghi & $1551.7 \mathrm{~b}$ & $66.9 \mathrm{fg}$ & $11,806.7$ def & 1275.7 cdefg & 326.7 cde \\
\hline $01 / 1273$ & $1423.3 \mathrm{bcd}$ & 1167.7 cdef & $116.8 \mathrm{efg}$ & $16,590.0 \mathrm{~b}$ & $1617.3 \mathrm{abc}$ & $275.8 \mathrm{fg}$ \\
\hline $01 / 1277$ & 1250.3 defg & 1237.7 cde & $658.4 \mathrm{ab}$ & 10,783.3 efghi & 1410.0 bcdef & 199.8 ij \\
\hline $01 / 1331$ & 1128.0 defgh & 1026.7 efg & 200.0 cdefg & $14,720.0 \mathrm{bc}$ & 1292.3 cdefg & $270.0 \mathrm{fgh}$ \\
\hline $01 / 1335$ & 1309.0 cde & $878.3 \mathrm{fgh}$ & $446.6 \mathrm{abcd}$ & $14,796.7 \mathrm{bc}$ & $1993.7 \mathrm{a}$ & $426.7 \mathrm{a}$ \\
\hline $01 / 1368$ & 830.0 hij & $1410.7 \mathrm{bc}$ & $676.2 \mathrm{a}$ & 8693.3 ghi & $996.3 \mathrm{gh}$ & $282.7 \mathrm{efg}$ \\
\hline $01 / 1371$ & $1424.3 \mathrm{bcd}$ & $1342.0 \mathrm{bcd}$ & 403.9 abcde & $12,290.0 \mathrm{cdef}$ & $813.3 \mathrm{~h}$ & $311.3 \mathrm{cdef}$ \\
\hline $01 / 1412$ & $1742.3 \mathrm{ab}$ & $1401.0 \mathrm{bc}$ & $131.9 \mathrm{efg}$ & $12,636.7$ cde & $807.7 \mathrm{~h}$ & 289.9 ef \\
\hline $01 / 1413$ & 1303.3 cdef & $930.0 \mathrm{fgh}$ & $77.2 \mathrm{fg}$ & $12,303.3$ cdef & $847.7 \mathrm{~h}$ & 300.3 def \\
\hline $01 / 1442$ & 939.0 ghij & 1047.0 defg & 256.7 cdefg & 10,556.7 efghi & $801.0 \mathrm{~h}$ & $272.7 \mathrm{fg}$ \\
\hline $01 / 1610$ & 1005.3 efghi & $1941.0 \mathrm{a}$ & 356.8 bcdef & $11,543.3$ def & 1252.7 cdefg & 302.7 cdef \\
\hline $01 / 1646$ & 1153.0 defgh & $1347.7 \mathrm{bcd}$ & $61.3 \mathrm{fg}$ & $12,743.3$ cde & 1188.7 defgh & $271.3 \mathrm{fgh}$ \\
\hline $01 / 1649$ & 870.0 hij & $889.0 \mathrm{fgh}$ & $64.8 \mathrm{fg}$ & $13,930.0 \mathrm{bcd}$ & $1070.0 \mathrm{fgh}$ & 312.0 cdef \\
\hline $01 / 1663$ & 947.3 fghij & $1588.3 \mathrm{~b}$ & $83.6 \mathrm{fg}$ & $12,966.7 \mathrm{cde}$ & 1152.0 efgh & $377.3 \mathrm{~b}$ \\
\hline $90 / 01554$ & $1629.3 \mathrm{bc}$ & $1418.7 \mathrm{bc}$ & $650.5 \mathrm{ab}$ & 9586.7 fghi & 1317.7 cdefg & $264.2 \mathrm{fgh}$ \\
\hline $94 / 0006$ & $708.7 \mathrm{ij}$ & 1028.0 efg & $68.6 \mathrm{fg}$ & $12,736.7$ cde & $1563.7 \mathrm{bcd}$ & $351.7 \mathrm{bc}$ \\
\hline $94 / 0330$ & 1139.0 defgh & $1419.0 \mathrm{bc}$ & $44.0 \mathrm{~g}$ & 8206.7 i & 1298.7 cdefg & $222.8 \mathrm{hij}$ \\
\hline $95 / 0379$ & 827.0 hij & 1137.7 cdef & $72.5 \mathrm{fg}$ & $11,806.7 \mathrm{def}$ & $1125.3 \mathrm{efgh}$ & $238.3 \mathrm{ghi}$ \\
\hline 98/2132 & 883.3 hij & $1004.3 \mathrm{efgh}$ & 146.8 defg & $11,513.3 \mathrm{defg}$ & 1503.3 bcde & $273.7 \mathrm{fg}$ \\
\hline TME1(check) & $596.3 \mathrm{j}$ & $776.7 \mathrm{gh}$ & $68.9 \mathrm{fg}$ & 8323.3 hi & 1495.0 bcde & $204.2 \mathrm{ij}$ \\
\hline 91/02324(check) & 1096.0 defgh & $715.7 \mathrm{~h}$ & $459.0 \mathrm{abc}$ & $11,146.7 \mathrm{defg}$ & $1082.0 \mathrm{fgh}$ & $342.3 \mathrm{bcd}$ \\
\hline 30,572(check) & 1076.3 defgh & $893.7 \mathrm{fgh}$ & 97.1 efg & $10,626.7$ efghi & $1002.0 \mathrm{gh}$ & $184.6 \mathrm{j}$ \\
\hline
\end{tabular}

Mean values in the same column with the different letters are significantly different at $p<0.05$. Parameters were analyzed in duplicate and expressed in $\mathrm{mg} / \mathrm{kg}$.

From the results for trial 2 in Table 5, the study showed that genotype 01/1417 (2833.3 mg/kg) had the highest $\mathrm{Ca}$ content of all genotypes investigated. However, genotypes 01/1181 and 01/1206 showed a significantly higher Ca content than 30,572 (check) and 91/02324 (check). Although the highest $\mathrm{Mg}$ content was recorded in genotype $01 / 1417(1679.7 \mathrm{mg} / \mathrm{kg})$, its concentration was found to be the lowest in genotypes 01/1231 (753.7 mg/kg) and 01/1172 $(721.7 \mathrm{mg} / \mathrm{kg})$ from all the genotypes examined. The highest levels of $\mathrm{Na}$ content were recorded in genotype $01 / 1417(73.9 \mathrm{mg} / \mathrm{kg})$. The study revealed that the levels of $\mathrm{K}$ content in genotype $01 / 1206(23,000 \mathrm{mg} / \mathrm{kg})$ were higher than the rest of the genotypes. However, genotype $99 / 7558(12,680 \mathrm{mg} / \mathrm{kg})$ had the lowest levels of all the genotypes examined. For P, the study showed the highest concentration in the Z97/0474 (12,680 mg/kg) genotype of all the samples examined. However, genotypes 99/7558 (760 mg/kg) and 01/1296 (763.7 mg/kg) had the lowest levels of P content of all the genotypes. Genotype Z97/0474 (358 mg/kg) showed the highest levels of $S$ content of all the samples studied. From the results above, all the genotypes with significantly higher mineral concentrations than the check genotypes could be recommended for breeding purposes. However, genotypes with no significant difference or significantly lower mineral concentrations than the check genotypes could not be recommended for such purposes as breeding. 
Table 5. Macromineral contents of yellow-fleshed cassava roots (trial Set 2) by genotype.

\begin{tabular}{ccccccc}
\hline \multirow{2}{*}{ Genotype } & Ca & $\mathbf{M g}$ & $\mathbf{N a}$ & $\mathbf{K}$ & $\mathbf{P}$ & $\mathbf{S}$ \\
\cline { 2 - 7 } & \multicolumn{5}{c}{ Parameters $\mathbf{( m g} / \mathbf{k g})$} \\
\hline $00 / 0028$ & $1296.0 \mathrm{cdefgh}$ & $1319.3 \mathrm{bcde}$ & $42.8 \mathrm{cdef}$ & $19,180.0 \mathrm{abcde}$ & $1166.7 \mathrm{bcde}$ & $320.0 \mathrm{abc}$ \\
$01 / 1181$ & $1782.0 \mathrm{bc}$ & $1455.7 \mathrm{abc}$ & $54.8 \mathrm{abc}$ & $17,976.7 \mathrm{bcdef}$ & $1173.0 \mathrm{bcde}$ & $263.7 \mathrm{cdefg}$ \\
$01 / 1206$ & $1922.3 \mathrm{~b}$ & $1108.7 \mathrm{defg}$ & $18.4 \mathrm{f}$ & $23,000.0 \mathrm{a}$ & $1290.3 \mathrm{abc}$ & $206.4 \mathrm{gh}$ \\
Z97/0474 & $1464.3 \mathrm{bcdef}$ & $1465.7 \mathrm{ab}$ & $35.5 \mathrm{cdef}$ & $20,046.7 \mathrm{abcd}$ & $1460.7 \mathrm{a}$ & $358.0 \mathrm{a}$ \\
$99 / 2987$ & $1416.3 \mathrm{bcdefg}$ & $1460.7 \mathrm{ab}$ & $28.8 \mathrm{def}$ & $19,100.0 \mathrm{abcde}$ & $1219.3 \mathrm{abc}$ & $321.2 \mathrm{abc}$ \\
$01 / 1404$ & $1550.5 \mathrm{bcdef}$ & $1147.0 \mathrm{bcdefg}$ & $32.9 \mathrm{cdef}$ & $17,461.7 \mathrm{bcdef}$ & $1204.6 \mathrm{abc}$ & $225.5 \mathrm{fgh}$ \\
$01 / 1417$ & $2833.3 \mathrm{a}$ & $1679.7 \mathrm{a}$ & $73.9 \mathrm{a}$ & $21,630.0 \mathrm{ab}$ & $892.0 \mathrm{fg}$ & $338.3 \mathrm{ab}$ \\
$01 / 1380$ & $1069.7 \mathrm{fgh}$ & $1039.7 \mathrm{efgh}$ & $23.7 \mathrm{ef}$ & $19,016.7 \mathrm{abcde}$ & $1143.7 \mathrm{cdef}$ & $250.4 \mathrm{defgh}$ \\
$01 / 1635$ & $1603.0 \mathrm{bcde}$ & $1045.3 \mathrm{efgh}$ & $45.0 \mathrm{bcde}$ & $17,506.7 \mathrm{bcdef}$ & $1187.0 \mathrm{bcde}$ & $299.3 \mathrm{bcd}$ \\
$01 / 1659$ & $863.3 \mathrm{~h}$ & $1238.0 \mathrm{bcdef}$ & $19.6 \mathrm{f}$ & $20,850.0 \mathrm{abc}$ & $1303.7 \mathrm{abc}$ & $292.7 \mathrm{bcd}$ \\
$01 / 1423$ & $1151.0 \mathrm{efgh}$ & $857.3 \mathrm{gh}$ & $29.2 \mathrm{def}$ & $18,356.7 \mathrm{abcdef}$ & $930.3 \mathrm{defg}$ & $275.8 \mathrm{cdef}$ \\
$01 / 1560$ & $1393.0 \mathrm{cdefg}$ & $1126.7 \mathrm{cdefg}$ & $34.9 \mathrm{cdef}$ & $15,613.3 \mathrm{defg}$ & $884.3 \mathrm{fg}$ & $219.9 \mathrm{fgh}$ \\
$99 / 7558$ & $810.0 \mathrm{~h}$ & $895.7 \mathrm{gh}$ & $42.7 \mathrm{cdef}$ & $12,680.0 \mathrm{~g}$ & $760.0 \mathrm{~g}$ & $225.5 \mathrm{fgh}$ \\
$01 / 1296$ & $1594.0 \mathrm{bcdef}$ & $1430.0 \mathrm{abcd}$ & $48.8 \mathrm{bcd}$ & $19,603.3 \mathrm{abcd}$ & $763.7 \mathrm{~g}$ & $227.8 \mathrm{fgh}$ \\
$01 / 1231$ & $1217.3 \mathrm{defgh}$ & $753.7 \mathrm{~h}$ & $39.9 \mathrm{cdef}$ & $16,066.7 \mathrm{defg}$ & $1074.7 \mathrm{cdef}$ & $201.9 \mathrm{~h}$ \\
$01 / 1551$ & $1414.3 \mathrm{bcdefg}$ & $952.0 \mathrm{fgh}$ & $68.1 \mathrm{ab}$ & $14,263.3 \mathrm{fg}$ & $924.0 \mathrm{efg}$ & $286.7 \mathrm{bcde}$ \\
$01 / 1172$ & $914.3 \mathrm{gh}$ & $721.7 \mathrm{~h}$ & $28.6 \mathrm{def}$ & $16,513.3 \mathrm{cdefg}$ & $1126.7 \mathrm{cdef}$ & $288.7 \mathrm{bcd}$ \\
$91 / 02324$ (check) & $1708.0 \mathrm{bcd}$ & $835.0 \mathrm{gh}$ & $53.0 \mathrm{abcd}$ & $16,123.3 \mathrm{defg}$ & $1438.0 \mathrm{ab}$ & $319.3 \mathrm{abc}$ \\
30,572 (check) & $1446.7 \mathrm{bcdef}$ & $932.3 \mathrm{fgh}$ & $23.8 \mathrm{ef}$ & $14,800.0 \mathrm{efg}$ & $1202.0 \mathrm{abcd}$ & $230.4 \mathrm{efgh}$ \\
\hline
\end{tabular}

Mean values in the same column with the different letters are significantly different at $p<0.05$. Parameters were analysed in duplicate and expressed in $\mathrm{mg} / \mathrm{kg}$.

From these results, it could be inferred that most of the genotypes had higher levels of mineral density than the check genotypes, and they could be advanced to the next stage in the breeding program. Additionally, there were significant differences in the macroelement concentrations among the genotypes. Most of the values obtained for the macroelements for all the genotypes compared well with what most researchers reported. Chiwona-Karltun et al. [19] reported the macroelement content of the nine cassava varieties as follows: Potassium content ranged from 6810 to $12,200 \mathrm{mg} / \mathrm{kg}$, and sodium ranged from 1720 (Nalumino variety) to $4590 \mathrm{mg} / \mathrm{kg}$ (Bangweulu variety). Otache et al. [20] reported that the macroelements of the peel of the three cassava cultivars studied was a calcium content of 137.4 to $198.1 \mathrm{mg} / \mathrm{kg}$ ), sodium of 137.0 to $142.3 \mathrm{mg} / \mathrm{kg}$, magnesium of 128.0 to $148.1 \mathrm{mg} / \mathrm{kg}$, and potassium of 787.2 to $1239.8 \mathrm{mg} / \mathrm{kg}$. Besides, the authors also corroborated the finding from this study that cassava roots are rich in macroelements, especially potassium. However, the values of macroelements reported by Afoakwa et al. [21] for six varieties obtained from the Crop Research Institute of Council for Scientific and Industrial Research (CSIR) in Ghana were lower than those obtained in this study and reported. The reported calcium content ranged from 0.06 to $1.60 \mathrm{mg} 100 \mathrm{~g}^{-1}$, sodium ranged from 0.25 to $0.37 \mathrm{mg}$

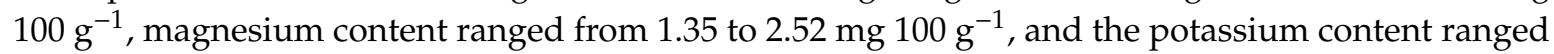
from 0.25 to $0.36 \mathrm{mg} 100 \mathrm{~g}^{-1}$. The difference in the values could be due to the genotypic differences and the environment. This study used yellow-fleshed genotypes, but most of the authors have used white-fleshed varieties. This implies that yellow-fleshed varieties are rich in macroelements compared with white-fleshed varieties. Additionally, it was observed in this study that most of the genotypes had the lowest sodium content among the macroelements, which was not in agreement with what some authors (Aro et al. [16]; Afoakwa et al. [21]; Otache et al. [20]) reported for white-fleshed varieties. This could also be due to genetic variation in terms of yellow-fleshed versus white-fleshed varieties. However, Chavez et al. [22] and Manano et al. [17] reported sodium as the lowest macroelement in cassava roots, as found in this study, which reported a sodium content of 3.6 to $3.9 \mathrm{mg} 100 \mathrm{~g}^{-1}$.

\subsection{Micromineral Concentrations of Yellow Cassava Roots by Genotype}

The means of the microminerals concentrations of yellow cassava roots (Trial 1) by genotype are shown in Table 6. There were significant differences $(p 0.05)$ on the levels of minerals that existed amongst the cassava varieties. Genotype 01/1235 (13.033 mg/kg) had the highest Fe concentration compared to the rest of the samples of cassava genotypes investigated. However, the Fe contents 
were the lowest in the check genotype 91/02324 $(5.637 \mathrm{mg} / \mathrm{kg})$. On the other hand, its concentrations were significantly higher in genotypes $01 / 1662,01 / 1412$, and $01 / 1610$ compared to those found in the check genotypes (TME, 91/02324, and 30,572). Although genotypes 01/1662 (24.414 mg/kg), 01/1273 $(24.800 \mathrm{mg} / \mathrm{kg})$, and $01 / 1335(24.823 \mathrm{mg} / \mathrm{kg})$ had the highest Mn contents, its concentration was found to be the lowest in genotypes $95 / 03379(9.544 \mathrm{mg} / \mathrm{kg})$. On the other hand, genotypes $01 / 1224,01 / 1663$, 90/01554, 98/2135, 94/0330, 01/1413, 01/1331, 01/1277, and 01/1235 showed significantly higher contents of Mn than all the check genotypes. Genotype 01/1335 $(2.698 \mathrm{mg} / \mathrm{kg})$ showed the highest levels of B as compared to the rest of the samples examined. Its concentration was also found to be significantly higher in genotypes $01 / 1662,01 / 1273,01 / 1277,01 / 1331,01 / 1442,01 / 1649,90 / 01554$, and 95/0379. However, the rest of the genotypes showed the least concentrations of B. Genotype 01/1662 (5.504 mg/kg) showed the highest levels of $\mathrm{Cu}$ as compared to the rest of the cassava genotypes investigated. However, its contents were the lowest in genotype 01/1235 $(1.745 \mathrm{mg} / \mathrm{kg})$. On the other hand, Cu contents were found to be significantly higher in genotype 98/2132. All the genotypes had significant amounts of Co and were significantly the same with the checks. For Ni, the highest levels were recorded in genotype $01 / 1273(3.449 \mathrm{mg} / \mathrm{kg})$. However, its levels were the lowest in genotype 01/1412 $(1.449 \mathrm{mg} / \mathrm{kg})$. On the other hand, genotype 01/1335 showed significantly higher levels of $\mathrm{Ni}$ than what was found in the checks. Zinc (Zn) had the highest levels in genotype $01 / 1115(11.523 \mathrm{mg} / \mathrm{kg})$. The results of the study also showed that genotypes 01/1273, 01/1442, 95/0379, 01/1662, and 01/1371 had significantly higher levels of $\mathrm{Zn}$ than those found in check genotypes.

Table 6. Means of the micromineral concentrations of yellow cassava roots (trial set 1) by genotype.

\begin{tabular}{|c|c|c|c|c|c|c|c|c|c|}
\hline \multirow{2}{*}{ Genotype } & $\mathrm{Fe}$ & Mn & B & $\mathrm{Cu}$ & Mo & Co & $\mathrm{Ni}$ & Zn & Al \\
\hline & \multicolumn{9}{|c|}{ Parameters (mg/kg) } \\
\hline $01 / 1662$ & $10.799 \mathrm{ab}$ & $24.414 \mathrm{a}$ & $2.383 \mathrm{ab}$ & $5.504 \mathrm{a}$ & $0.700 \mathrm{a}$ & $0.700 \mathrm{a}$ & 2.044 efghij & $9.789 \mathrm{abc}$ & $5.000 \mathrm{ab}$ \\
\hline $01 / 1224$ & 8.691 bcdef & $19.011 \mathrm{abc}$ & $2.000 \mathrm{~b}$ & 3.918 bcdef & $0.700 \mathrm{a}$ & $0.663 \mathrm{a}$ & 2.164 defghi & 7.939 bcdef & $5.000 \mathrm{ab}$ \\
\hline $01 / 1235$ & $13.033 \mathrm{a}$ & 17.869 abcdef & $2.032 \mathrm{~b}$ & $1.745 \mathrm{~h}$ & $0.700 \mathrm{a}$ & $0.688 \mathrm{a}$ & 1.784 ghij & 7.806 bcdef & $6.050 \mathrm{a}$ \\
\hline $01 / 1273$ & 9.749 abcde & $24.800 \mathrm{a}$ & $2.449 \mathrm{ab}$ & $4.569 \mathrm{abc}$ & $0.675 \mathrm{ab}$ & $0.675 \mathrm{a}$ & $3.449 \mathrm{a}$ & $10.303 \mathrm{ab}$ & $4.750 \mathrm{ab}$ \\
\hline $01 / 1277$ & 7.016 bcdef & 17.115 abcdef & $2.210 \mathrm{ab}$ & $2.268 \mathrm{gh}$ & $0.700 \mathrm{a}$ & $0.688 a$ & $2.994 \mathrm{abc}$ & 7.743 bcdef & $5.000 \mathrm{ab}$ \\
\hline $01 / 1368$ & 7.965 bcdef & 10.376 def & $2.000 \mathrm{~b}$ & 3.069 defgh & $0.675 \mathrm{ab}$ & $0.650 \mathrm{a}$ & 2.065 efghij & 8.826 bcde & $4.750 \mathrm{ab}$ \\
\hline 01/1371 & 6.878 cdef & 13.600 bcdef & $2.103 \mathrm{~b}$ & 3.873 bcdef & $0.700 \mathrm{a}$ & $0.663 \mathrm{a}$ & 2.654 bcde & $9.909 \mathrm{abc}$ & $5.000 \mathrm{ab}$ \\
\hline 01/1412 & $10.256 \mathrm{abcd}$ & 15.101 bcdef & $2.178 \mathrm{~b}$ & $2.369 \mathrm{gh}$ & $0.700 \mathrm{a}$ & $0.688 \mathrm{a}$ & $1.425 \mathrm{j}$ & $10.010 \mathrm{abc}$ & $5.200 \mathrm{ab}$ \\
\hline $01 / 1413$ & 9.819 abcde & 17.761 abcdef & $2.089 \mathrm{~b}$ & 3.350 cdefg & $0.700 \mathrm{a}$ & $0.675 \mathrm{a}$ & 2.566 bcdef & $9.228 \mathrm{abcd}$ & $5.000 \mathrm{ab}$ \\
\hline $01 / 1442$ & 8.898 abcdef & 13.846 bcdef & $2.380 \mathrm{ab}$ & 4.175 abcde & $0.704 \mathrm{a}$ & $0.678 \mathrm{a}$ & 1.802 ghij & $10.214 \mathrm{ab}$ & $5.012 \mathrm{ab}$ \\
\hline $01 / 1610$ & $10.706 \mathrm{abc}$ & 15.574 bcdef & $2.115 \mathrm{~b}$ & 3.360 cdefg & $0.675 \mathrm{ab}$ & $0.650 \mathrm{a}$ & 2.409 bcdefgh & $9.366 \mathrm{abcd}$ & $4.750 \mathrm{ab}$ \\
\hline $94 / 0006$ & 7.888 bcdef & 9.726 ef & $2.071 \mathrm{~b}$ & $4.589 \mathrm{abc}$ & $0.663 \mathrm{~b}$ & $0.638 \mathrm{a}$ & 2.576 bcdef & 8.838 bcde & $4.500 \mathrm{~b}$ \\
\hline $94 / 0330$ & 7.353 bcdef & 17.950 abcde & $2.050 \mathrm{~b}$ & $2.339 \mathrm{gh}$ & $0.700 \mathrm{a}$ & $0.700 \mathrm{a}$ & 2.653 bcde & $9.411 \mathrm{abcd}$ & $5.000 \mathrm{ab}$ \\
\hline $95 / 0379$ & 7.800 bcdef & $9.544 \mathrm{f}$ & $2.218 \mathrm{ab}$ & $4.443 \mathrm{abcd}$ & $0.700 \mathrm{a}$ & $0.675 \mathrm{a}$ & 2.296 cdefgh & $10.238 \mathrm{ab}$ & $5.000 \mathrm{ab}$ \\
\hline $98 / 2132$ & 7.971 bcdef & 18.186 abcd & $2.088 \mathrm{~b}$ & $5.053 \mathrm{ab}$ & $0.700 \mathrm{a}$ & $0.675 \mathrm{a}$ & 2.854 abcd & 9.194 abcd & $5.000 \mathrm{ab}$ \\
\hline TME1(check) & 7.761 bcdef & 14.791 bcdef & $2.000 \mathrm{~b}$ & $2.606 \mathrm{fgh}$ & $0.700 \mathrm{a}$ & $0.688 \mathrm{a}$ & $1.551 \mathrm{ij}$ & $5.933 \mathrm{f}$ & $5.025 \mathrm{ab}$ \\
\hline 91/02324(check) & $5.637 \mathrm{f}$ & 13.356 bcdef & $2.007 \mathrm{~b}$ & 4.158 abcde & $0.700 \mathrm{a}$ & $0.676 \mathrm{a}$ & 1.708 hij & 7.710 bcdef & $4.999 \mathrm{ab}$ \\
\hline 30572(check) & 6.116 ef & 12.750 cdef & $2.000 \mathrm{~b}$ & 3.005 efgh & $0.688 \mathrm{ab}$ & $0.688 \mathrm{a}$ & 2.165 defghi & $6.345 \mathrm{ef}$ & $4.875 \mathrm{ab}$ \\
\hline
\end{tabular}

Mean values in the same column with different letters are significantly different at $p<0.05$. Parameters were analysed in duplicate and expressed in $\mathrm{mg} / \mathrm{kg}$.

The means of the micromineral concentrations of yellow cassava roots (Trial 2) by genotype are shown in Table 7. There were significant differences in the microelement concentrations among the genotypes. Genotype 00/0028 (14.851 mg/kg) had the highest Fe concentration as compared to the rest of the cassava genotypes investigated. However, the Fe contents were found to be the lowest in the check genotype $91 / 02324(5.539 \mathrm{mg} / \mathrm{kg})$. Additionally, genotype 00/0028 $(21.261 \mathrm{mg} / \mathrm{kg})$ had the highest Mn content, while its concentration was found to be the lowest in genotypes 99/7558 (8.850 mg/kg) and $01 / 1172(8.563 \mathrm{mg} / \mathrm{kg})$. The study also revealed that genotypes $00 / 0028(3.418 \mathrm{mg} / \mathrm{kg})$ and $01 / 1181$ $(3.366 \mathrm{mg} / \mathrm{kg})$ had the highest contents of B than the rest of the genotypes examined. However, its lowest concentrations were recorded in genotypes 01/1423 $(2.105 \mathrm{mg} / \mathrm{kg}), 99 / 7558(2.114 \mathrm{mg} / \mathrm{kg})$, 
01/1231 (2.106 mg/kg), 01/1551 (2.106 mg/kg), and check 91/0232 (2.107 mg/kg). For Cu, the highest concentrations were recorded in the check genotype $30,572(8.131 \mathrm{mg} / \mathrm{kg})$. However, its contents were the lowest in genotypes 01/1296 (3.061 mg/kg) and 01/1172 $(3.326 \mathrm{mg} / \mathrm{kg})$. All the genotypes recorded significant contents of both Mo and Co and were significantly the same with the checks. Nickel (Ni) had the highest concentration in genotype $00 / 0028(3.325 \mathrm{mg} / \mathrm{kg})$. All the genotypes showed significantly higher levels of the mineral concentration than the check genotypes, which are white-fleshed. Thus, the yellow-fleshed roots are richer in minerals than the white-fleshed roots. Besides, the genotypes that showed higher levels of mineral density than the check genotypes could be advanced to the next stage in the breeding program. Most of the values obtained for the microelements for all the genotypes compared well with what most researchers reported. Burns et al. [23] found that the concentrations of iron in tubers from all cultivars studied (range from $8-24 \mathrm{mg} / \mathrm{kg}$ ) were at the low end of the published range for cassava (3-140 mg/kg). In contrast, the range of tuber zinc concentrations, $8-19 \mathrm{mg} / \mathrm{kg}$, straddled the published standard for cassava of $14 \mathrm{mg} / \mathrm{kg}$ [23].

Table 7. Micromineral content of yellow cassava roots (trial set 2) by genotype.

\begin{tabular}{|c|c|c|c|c|c|c|c|c|c|}
\hline \multirow{2}{*}{ Genotype } & $\mathrm{Fe}$ & Mn & B & $\mathrm{Cu}$ & Mo & Co & $\mathrm{Ni}$ & Zn & Al \\
\hline & \multicolumn{9}{|c|}{ Parameters (mg/kg) } \\
\hline $00 / 0028$ & $14.851 \mathrm{a}$ & $21.261 \mathrm{a}$ & $3.418 \mathrm{a}$ & $5.040 \mathrm{abc}$ & $0.837 \mathrm{a}$ & $0.812 \mathrm{a}$ & $3.325 \mathrm{a}$ & 10.736 bcd & $5.375 \mathrm{~b}$ \\
\hline $01 / 1181$ & $14.465 \mathrm{ab}$ & 14.576 cde & $3.366 \mathrm{a}$ & $4.666 \mathrm{bc}$ & $0.912 \mathrm{a}$ & $0.912 \mathrm{a}$ & 2.003 bcde & 10.013 bcde & $7.281 \mathrm{ab}$ \\
\hline $01 / 1206$ & 8.683 bcde & $19.555 \mathrm{ab}$ & $2.979 \mathrm{ab}$ & $4.601 \mathrm{bc}$ & $0.775 \mathrm{a}$ & $0.775 \mathrm{a}$ & $1.436 \mathrm{de}$ & $16.036 \mathrm{a}$ & $5.852 \mathrm{ab}$ \\
\hline Z97/0474 & 10.116 abcde & $15.940 \mathrm{bc}$ & $2.674 \mathrm{ab}$ & $6.732 \mathrm{ab}$ & $0.675 \mathrm{a}$ & $0.675 \mathrm{a}$ & $2.511 \mathrm{abc}$ & $11.971 \mathrm{~b}$ & $4.750 \mathrm{~b}$ \\
\hline $99 / 2987$ & $11.505 \mathrm{abcd}$ & 15.133 bcde & $2.585 \mathrm{ab}$ & $5.636 \mathrm{abc}$ & $0.750 \mathrm{a}$ & $0.750 \mathrm{a}$ & 1.978 bcde & 9.999 bcde & $5.375 \mathrm{~b}$ \\
\hline $01 / 1404$ & 7.343 de & 15.118 bcde & $2.831 \mathrm{ab}$ & $5.012 \mathrm{abc}$ & $0.751 \mathrm{a}$ & $0.750 \mathrm{a}$ & 1.674 cde & 9.661 bcdefg & $5.711 \mathrm{ab}$ \\
\hline $01 / 1417$ & 9.109 abcde & $12.846 \mathrm{cdef}$ & $2.684 \mathrm{ab}$ & $3.929 b c$ & $0.675 \mathrm{a}$ & $0.650 \mathrm{a}$ & $2.203 \mathrm{bcd}$ & $11.505 \mathrm{bc}$ & $4.750 \mathrm{~b}$ \\
\hline $01 / 1380$ & $6.508 \mathrm{de}$ & $10.510 \mathrm{def}$ & $2.729 \mathrm{ab}$ & $6.186 \mathrm{abc}$ & $0.725 \mathrm{a}$ & $0.712 \mathrm{a}$ & $1.068 \mathrm{e}$ & 10.248 bcde & $6.297 \mathrm{ab}$ \\
\hline $01 / 1635$ & $13.218 \mathrm{abc}$ & $12.000 \mathrm{cdef}$ & $2.265 \mathrm{ab}$ & $3.890 \mathrm{bc}$ & $0.662 \mathrm{a}$ & $0.662 \mathrm{a}$ & $2.101 \mathrm{bcd}$ & 7.745 efgh & $4.625 \mathrm{~b}$ \\
\hline $01 / 1659$ & 8.882 bcde & 14.891 bcde & $2.415 \mathrm{ab}$ & $5.274 \mathrm{abc}$ & $0.662 \mathrm{a}$ & $0.638 \mathrm{a}$ & 1.645 cde & 9.104 bcdefgh & $4.625 \mathrm{~b}$ \\
\hline $01 / 1423$ & 11.038 abcde & 13.353 cdef & $2.105 \mathrm{~b}$ & $4.159 \mathrm{bc}$ & $0.650 \mathrm{a}$ & $0.625 \mathrm{a}$ & 1.576 cde & $12.009 \mathrm{~b}$ & $4.500 \mathrm{~b}$ \\
\hline $01 / 1560$ & 8.347 cde & $15.453 \mathrm{bcd}$ & $2.230 \mathrm{ab}$ & $3.890 \mathrm{bc}$ & $0.650 \mathrm{a}$ & $0.650 \mathrm{a}$ & $2.309 \mathrm{bcd}$ & $7.711 \mathrm{efgh}$ & $4.500 \mathrm{~b}$ \\
\hline $99 / 7558$ & $7.336 \mathrm{de}$ & $8.850 \mathrm{f}$ & $2.114 \mathrm{~b}$ & $3.633 \mathrm{bc}$ & $0.687 \mathrm{a}$ & $0.675 \mathrm{a}$ & $2.079 \mathrm{bcd}$ & $7.010 \mathrm{fgh}$ & $4.875 \mathrm{~b}$ \\
\hline $01 / 1296$ & 7.693 cde & $11.360 \mathrm{cdef}$ & $2.363 \mathrm{ab}$ & $3.061 \mathrm{c}$ & $0.650 \mathrm{a}$ & $0.650 \mathrm{a}$ & $2.711 \mathrm{ab}$ & 8.170 defgh & $4.500 \mathrm{~b}$ \\
\hline $01 / 1231$ & 8.561 cde & $11.210 \mathrm{cdef}$ & $2.106 \mathrm{~b}$ & $4.373 \mathrm{bc}$ & $0.663 \mathrm{a}$ & $0.625 \mathrm{a}$ & 1.866 bcde & 9.875 bcdef & $4.500 \mathrm{~b}$ \\
\hline $01 / 1551$ & 7.414 de & $12.469 \mathrm{cdef}$ & $2.106 \mathrm{~b}$ & $3.665 \mathrm{bc}$ & $0.662 \mathrm{a}$ & $0.650 \mathrm{a}$ & 1.578 cde & 9.732 bcdef & $4.500 \mathrm{~b}$ \\
\hline $01 / 1172$ & 8.814 bcde & $8.563 \mathrm{f}$ & $2.254 \mathrm{ab}$ & $3.326 \mathrm{c}$ & $0.625 \mathrm{a}$ & $0.600 \mathrm{a}$ & $1.370 \mathrm{de}$ & $6.495 \mathrm{~h}$ & $4.250 \mathrm{~b}$ \\
\hline 91/02324 (check) & $5.539 \mathrm{e}$ & $10.343 \mathrm{ef}$ & $2.107 \mathrm{~b}$ & $5.891 \mathrm{abc}$ & $0.675 \mathrm{a}$ & $0.675 \mathrm{a}$ & $2.433 a b c$ & $6.744 \mathrm{gh}$ & $4.865 \mathrm{~b}$ \\
\hline 30572 (check) & $11.376 \mathrm{abcd}$ & $10.841 \mathrm{def}$ & $2.479 \mathrm{ab}$ & $8.131 \mathrm{a}$ & $0.755 \mathrm{a}$ & $0.737 \mathrm{a}$ & $1.356 \mathrm{de}$ & 8.756 cdefgh & $9.379 \mathrm{a}$ \\
\hline
\end{tabular}

Mean values in the same column with different letters are significantly different at $p<0.05$. Parameters were analysed in duplicate and expressed in $\mathrm{mg} / \mathrm{kg}$.

\subsection{Effect of Maturity on the Macroelement and Microelement Contents of Yellow-Fleshed Cassava Roots}

Figure $1 \mathrm{a}, \mathrm{b}$ shows the concentrations of macrominerals in yellow-fleshed cassava roots by maturity (harvesting time) for both trials 1 and 2. For trial set 1, the study showed that there was a significant difference $(p<0.05)$ in the mean values for the Ca and P contents in cassava roots harvested at 9 and 12 months, respectively. However, the concentrations of $\mathrm{Ca}$ and $\mathrm{P}$ were higher in the cassava roots that were harvested at the age of 9 months than those at 12 months. Thus, harvesting time had a strong influence on the $\mathrm{Ca}$ and $\mathrm{P}$ content of cassava roots. However, there was no significant difference $(p>0.05)$ in the concentrations of $\mathrm{Mg}, \mathrm{Na}, \mathrm{K}$, and $\mathrm{S}$ of cassava genotypes harvested at 9 and 12 months. For trial 2, the study showed that there was a significant difference $(p<0.05)$ between the mean concentrations of $\mathrm{Ca}, \mathrm{Na}, \mathrm{K}, \mathrm{S}$, and $\mathrm{P}$ for cassava roots harvested at 9 and 12 months. However, the $\mathrm{Mg}$ concentration showed no difference in the mean value for cassava roots harvested at both maturity stages. The concentration of $\mathrm{Ca}, \mathrm{Na}, \mathrm{K}$, and $\mathrm{P}$ were higher in the cassava roots that were sampled at 9 months than those at 12 months except for $\mathrm{S}$, which was higher in the samples that matured at 12 months. It implies that harvesting time plays a major role in the concentration level of some macroelements in cassava roots while showing no effect on some. Additionally, it was established that harvesting time showed different effects on the two trials investigated. Thus, genotype plays a key role in the degree of the effects the harvesting time had on the macroelement concentrations of cassava roots. Richardson [23] evaluated the macroelements of cassava sample materials that were 
harvested at nine months after planting and reported that the values of sodium ranged from 22.3 to $34.9 \mathrm{mg} 100 \mathrm{~g}^{-1}$. In contrast, the values for potassium ranged from 817.3 to $1301.2 \mathrm{mg} 100 \mathrm{~g}^{-1}$ [24]. If compared with what was obtained in this study for cassava genotypes harvested at 9 months for both trials studied, the values of potassium in this study were higher and those of sodium lower. The difference could be due to the different genetic make-up of yellow-fleshed and white-fleshed cassava roots. This further confirmed that yellow-fleshed roots contain more macroelements than the white-fleshed roots, especially $\mathrm{K}$. However, the values of the macroelements obtained at 12 months for trial 2 compared well with what Chavez et al. [22] reported for cassava roots harvested at 10 to 11 months. He reported that samples had an average magnesium content of $1153 \mathrm{mg} / \mathrm{kg}$, sodium averaged at $66.4 \mathrm{mg} / \mathrm{kg}$, potassium averaged at $8903 \mathrm{mg} / \mathrm{kg}$, phosphorus averaged at $1284 \mathrm{mg} / \mathrm{kg}$, and sulphur averaged at $273 \mathrm{mg} / \mathrm{kg}$ dry weight. However, the macroelement values for trial 1 at 12 months showed higher values than what was reported in the literature [22].

Figure 1c,d shows the levels of microelements in yellow-fleshed cassava roots by maturity (harvesting time) for both trial sets 1 and 2. For trial set 1, the study showed that there was a significant difference $(p<0.05)$ in the concentration of $\mathrm{Fe}, \mathrm{Mn}, \mathrm{B}$, and $\mathrm{Ni}$ at different maturity levels. For $\mathrm{Fe}, \mathrm{Mn}$, and $\mathrm{B}$, higher concentrations were found in cassava roots that were harvested at 9 months. However, higher contents of $\mathrm{Ni}$ were recorded in cassava roots that were harvested at 12 months. The results of the study also revealed that there was no significant difference $(p>0.05)$ in the contents of $\mathrm{Cu}$, $\mathrm{Mo}, \mathrm{Co}, \mathrm{Zn}$, and $\mathrm{Al}$, regardless of the maturity stage. Thus, harvesting time had a strong influence on the contents of the minerals in cassava roots. For trial set 2, the study showed that there was a significant difference $(p<0.05)$ in the concentration of $\mathrm{Fe}, \mathrm{Mn}, \mathrm{B}, \mathrm{Ni}, \mathrm{Zn}$, and $\mathrm{Al}$ at different maturity stages. The concentrations of $\mathrm{Fe}, \mathrm{B}, \mathrm{Ni}$, and $\mathrm{Al}$ were found to be higher in the cassava roots that were harvested at 9 months. However, $\mathrm{Mn}$ and $\mathrm{Zn}$ contents were higher in cassava roots that were harvested at 12 months. The study further revealed that there was no significant difference $(p>0.05)$ in the contents of $\mathrm{Cu}, \mathrm{Mo}$, and $\mathrm{Co}$ regardless of the maturity stage. It implies that harvesting time plays a significant role in the concentration level of some microelements in the cassava roots while showing no effect on some. Additionally, it was established that harvesting time showed different effects on the two set trials investigated. Thus, genotype plays a vital role in the degree of effects the harvesting time showed on the microelement's concentrations of cassava roots.

\subsection{Effect of the Method of Sampling on the Distribution of the Macro- and Microelement Contents of Yellow-Fleshed Cassava Roots}

The mean values of the $\mathrm{Ca}, \mathrm{Mg}, \mathrm{Na}, \mathrm{K}, \mathrm{P}$, and $\mathrm{S}$ content in yellow root cassava genotypes by sampling methods-method $1(\mathrm{ABC})$ and method $2(\mathrm{~L})$ are presented in Figure 2a,b. The table also gives information on the distribution of the macroelements from the proximal, middle, and distal parts of cassava roots. There was no significant $(p>0.05)$ difference in the mean values of all macroelements studied in the proximal and middle parts of cassava roots except $\mathrm{S}$, which showed a significant $(p<0.05)$ difference. Additionally, there were significant $(p<0.05)$ differences between the mean values of all the macroelements from the distal part and that of the proximal or middle part. It implies that macroelement concentrations are evenly distributed from the proximal to middle parts, but from the middle to the distal part, they are not evenly distributed. A similar pattern was observed for K, $\mathrm{P}$, and $\mathrm{S}$ for set trial 2. However, a slightly different pattern was observed for the mean values of $\mathrm{Ca}$, $\mathrm{Mg}$, and $\mathrm{Na}$, where there was no significant $(p<0.05)$ difference observed for the proximal, middle, and distal sections of cassava roots. The results showed that the distribution of macroelements within the cassava is mineral dependent. It was also observed that the macroelement concentrations were lower at the distal part than the proximal or middle section. This could be due to the dilution effect because it has been established that the water distribution of cassava increases from the proximal to distal parts. However, we could conclude that the best part to sample macroelements in cassava roots should be from the proximal or middle part. The two methods were compared by averaging the values of each of the macroelements from the proximal, middle, and distal parts (method 1) and 
comparing them with the values of the macroelements from the longitudinal section of the cassava roots (method 2).
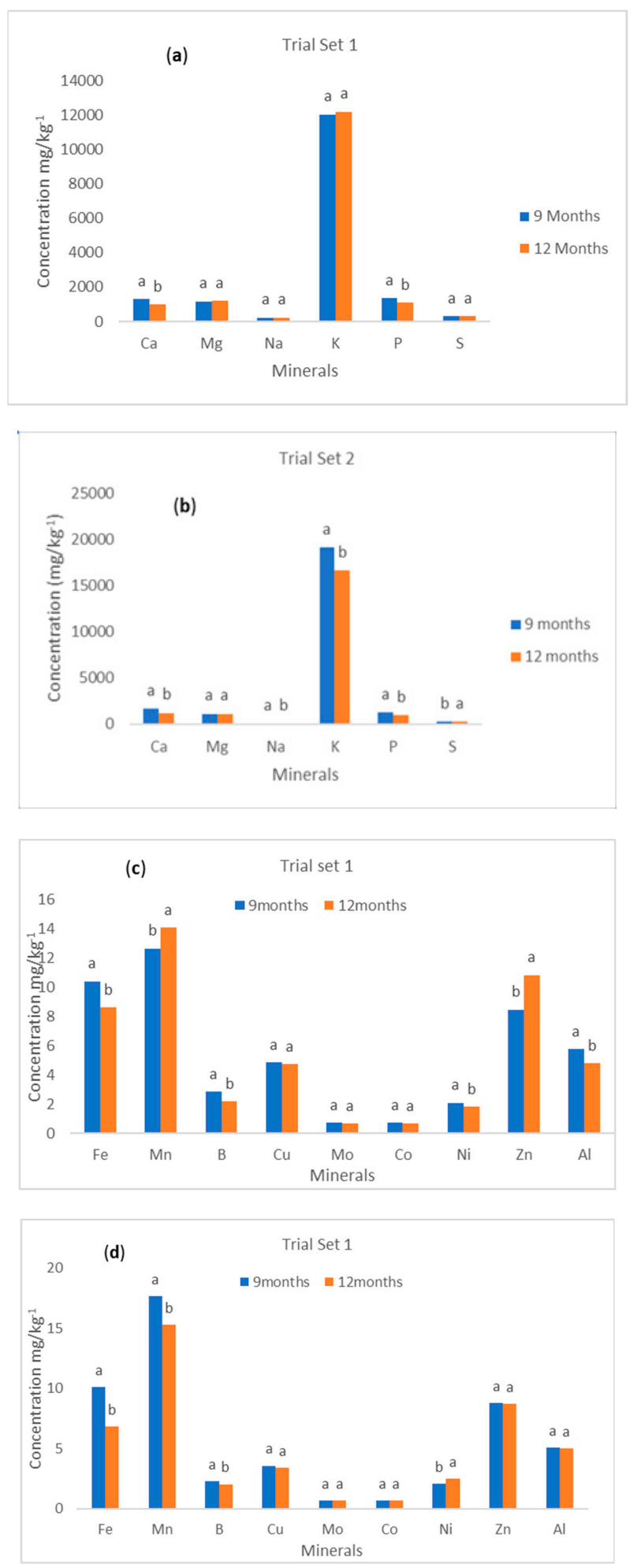

Figure 1. (a,b) Macromineral content of yellow-fleshed roots (trial sets 1 and 2) by maturity. Mean values with different letters are significantly different at $p<0.05$. (c,d) Micromineral content of yellow-fleshed cassava roots (trial sets 1 and 2) by maturity. Mean values with different letters are significantly different at $p<0.05$. 

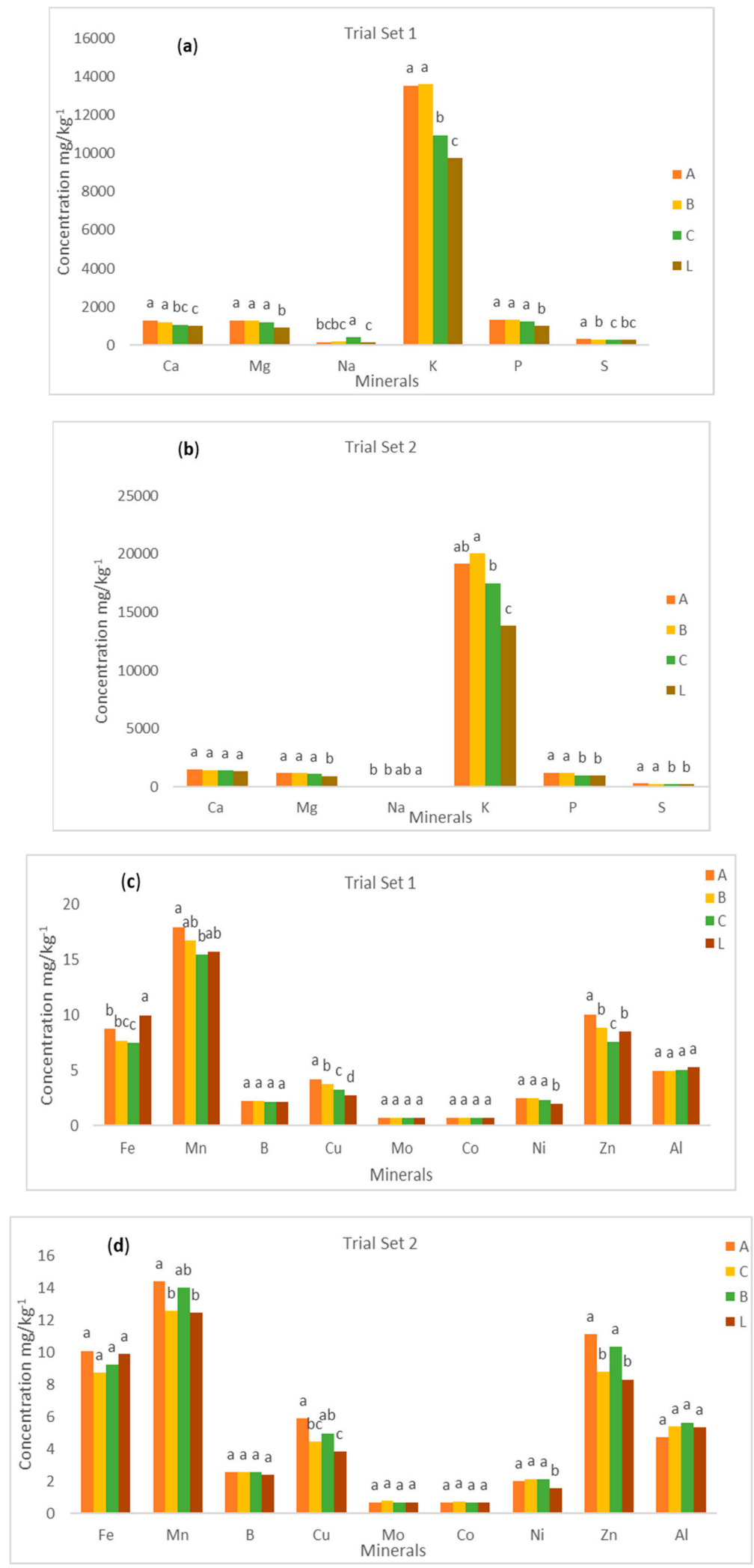

Figure 2. (a,b) Macromineral content of yellow-fleshed cassava roots (trial sets 1 and 2) by sampling methods. Mean values with different letters are significantly different at $p<0.05$; $\mathrm{A}=$ Proximal; B = Middle; C = Distal; L = Longitudinal. (c,d) Micromineral content of yellow-fleshed cassava roots (trial sets 1 and 2 ) by sampling methods. Mean values with different letters are significantly different at $p<0.05 ; \mathrm{A}=$ Proximal; $\mathrm{B}=$ Middle; $\mathrm{C}=$ Distal $\mathrm{L}=$ Longitudinal. 
For both trials 1 and 2, there was a significant $(p<0.05)$ difference in the mean values of all the macroelements except the Ca content of the set 2 trial. This shows that the concentration of the macroelements as a method is sampling dependent. It was observed that the mean value of all macroelements from method 1 was significantly $(p<0.05)$ higher than the mean values from method 2 for both trials 1 and 2 . The levels of $\mathrm{Ca}, \mathrm{Mg}, \mathrm{K}$, and $\mathrm{P}$ were significantly higher in the proximal and middle portions of the samples in method ABC (method 1) than method L (method 2). However, the levels of $\mathrm{Na}$ and $\mathrm{S}$ were far higher in the distal and proximal portions in method $\mathrm{ABC}$ than method $\mathrm{L}$, respectively. From this result, method 1 (where samples were taken from all parts and averaged) was found to be the best, but it is not cost-effective. Method 2 could be the best alternative because it is cost-effective but not as accurate as method 1. It can be recommended that using sampling method 1 to determine $\mathrm{Ca}, \mathrm{Mg}, \mathrm{Na}, \mathrm{K}, \mathrm{P}$, and $\mathrm{S}$ contents in yellow root cassava is better than using method 2 as it gives a far higher value of the mineral content. For set trial 1, the study showed that there was a significant $(p<0.05)$ difference in the mean values of macroelements. The levels of $\mathrm{Ca}, \mathrm{Mg}, \mathrm{K}$, and $\mathrm{P}$ were significantly higher in the proximal and middle portions of the samples in method $A B C$ than method L. However, the levels of $\mathrm{Na}$ and $\mathrm{S}$ were far higher in the distal and proximal portions in method $\mathrm{ABC}$ than method $\mathrm{L}$, respectively. From this result, it can be recommended that using sampling method $\mathrm{ABC}$ to extract $\mathrm{Ca}, \mathrm{Mg}, \mathrm{Na}, \mathrm{K}, \mathrm{P}$, and $\mathrm{S}$ contents in yellow root cassava is better than using method $\mathrm{L}$ as it gives a far higher value of the mineral content. This result was similar to Maziya-Dixon et al. [13]. They observed that the mean cis- and trans-beta-carotene contents were generally highest in the proximal end and lowest in the distal end of yellow-fleshed cassava storage roots. However, they reported that there was no significant difference $(p>0.05)$ between the two methods (the sectional and longitudinal) for the cis- and trans-beta-carotene content. The contrary results indicate that micronutrients are sampling dependent. The sampling method for minerals could be different for carotenes.

The mean values of $\mathrm{Fe}, \mathrm{Mn}, \mathrm{B}, \mathrm{Cu}, \mathrm{Mo}, \mathrm{Co}, \mathrm{Ni}, \mathrm{Zn}$, and $\mathrm{Al}$ contents in yellow root cassava genotypes by sampling methods-method 1 (ABC) and method $2(\mathrm{~L})$-are presented in Figure 2c,d. The figure also gives information on the distribution of the microelements from the proximal, middle, and distal parts of cassava roots. From trial set 1 , there was a significant difference $(p<0.05)$ in the mean values of $\mathrm{Fe}, \mathrm{Mn}, \mathrm{Cu}$, and $\mathrm{Zn}$ elements in all the three sections (proximal, middle, and distal) of cassava root. A similar pattern was observed for $\mathrm{Mn}$, and $\mathrm{Cu}$ in the trial set 2 . The mean values for $\mathrm{Zn}$ showed a significant difference in the proximal and middle parts only. This result implies that the concentrations of these microelements are not evenly distributed from the proximal to the distal part of the cassava root. However, the study showed that there was no significant difference $(p>0.05)$ in the mean values of $\mathrm{B}, \mathrm{Mo}, \mathrm{Co}, \mathrm{Ni}$, and $\mathrm{Al}$ elements in the three sections of cassava root. Similarly, the mean values for $\mathrm{Fe}, \mathrm{B}, \mathrm{Mo}, \mathrm{Co}, \mathrm{Ni}$, and $\mathrm{Al}$ did not show any significant difference $(p>0.05)$ in all three sections in the trial set 2 . Further, the study revealed that there was no significant difference $(p>0.05)$ in the mean values of all microelements in the proximal part of the cassava root. It implies that the concentrations of $\mathrm{Fe}, \mathrm{B}, \mathrm{Mo}, \mathrm{Co}, \mathrm{Ni}$, and $\mathrm{Al}$ are evenly distributed across the three cassava root sections that were studied.

The results showed that the distribution of microelements in the cassava root is mineral dependent. It was also observed that the microelement concentrations were lower in the distal part than the proximal or middle section. This could be due to the dilution effect because it has been established that the water distribution of cassava increases from the proximal to the distal part. However, we could conclude that the best part to sample microelements in cassava roots from is the proximal or middle part. The two sampling methods were compared by averaging the values of each of the microelements from the proximal, middle, and distal parts (method 1) and comparing them with the values of the microelements from the longitudinal section of the cassava roots (method 2). For both trial 1 and 2, there was a significant $(p<0.05)$ difference in the mean values of all the microelements except the $\mathrm{B}$, $\mathrm{Mo}, \mathrm{Co}$, and $\mathrm{Al}$ content of trial set 1 . Additionally, there was a significant difference $(p<0.05)$ in the mean values for all the microelements except for $\mathrm{Fe}, \mathrm{B}, \mathrm{Mo}, \mathrm{Co}$, and $\mathrm{Al}$ in the trial set 2 . This shows 
that the concentration of microelements in a method that is sampling dependent. It was observed that the mean value of all microelements from method 1 was significantly $(p<0.05)$ higher than the mean values from method 2 for both trial set 1 and 2. The levels of $\mathrm{Mn}, \mathrm{Cu}, \mathrm{Ni}$, and $\mathrm{Zn}$ were found to be significantly higher in the proximal portions of the samples in method ABC (method 1) than method $\mathrm{L}$ (method 2). However, the study showed that Fe had far higher levels in method L (method 2) than method ABC (method 1$)$ in the trial set 1.

Further, the results of the study revealed that the $\mathrm{B}, \mathrm{Mo}, \mathrm{Co}$, and $\mathrm{Al}$ contents did not show any significant difference $(p>0.05)$ between the two sampling methods. For trial set 2 , the contents of $\mathrm{Mn}, \mathrm{Cu}, \mathrm{Ni}$, and $\mathrm{Zn}$ were found to be significantly higher in the proximal portions of the samples investigated in method ABC (method 1) than in method L (method 2). However, the study revealed that the levels of $\mathrm{Fe}, \mathrm{B}, \mathrm{Mo}, \mathrm{Co}$, and $\mathrm{Al}$ did not show any significant difference in the two sampling methods. As a result, any of the two methods could be used for the extraction of these microelements. It can also be recommended that using sampling method $\mathrm{ABC}$ (method 1) for extraction of $\mathrm{Mn}, \mathrm{Cu}, \mathrm{Ni}$, and $\mathrm{Zn}$ contents in cassava roots is better than using method L (method 2).

\subsection{Pearson Correlation Coefficients of Macro- and Micromineral Contents of Yellow-Fleshed Cassava Roots}

The Pearson correlation coefficients of the macromineral content in yellow root cassava samples investigated are presented in Table 8 . From the dataset of trial 1, there was a significant positive $(p<0.001)$ correlation between the $\mathrm{K}$ and $\mathrm{P}$ content $(\mathrm{r}=0.513)$. This correlation shows the possibility of breeding yellow root cassava varieties with high $\mathrm{K}$ and $\mathrm{P}$ mineral contents. However, the results showed that there was a significant negative $(p<0.05)$ correlation between the $\mathrm{Na}$ and $\mathrm{K}$ content $(\mathrm{r}=-0.271)$. Further, a significant $(p<0.05)$ but weak correlation was observed between the $\mathrm{P}$ and $\mathrm{Mg}$ content $(\mathrm{r}=-0.062), \mathrm{P}$ and Na content $(\mathrm{r}=-0.073)$, and $\mathrm{S}$ and $\mathrm{Na}$ content $(\mathrm{r}=-0.67)$. From the dataset of trial 2, the results showed a significant positive $(p<0.001)$ correlation between the Ca and Mg content $(r=0.605), C a$ and $K$ content $(r=0.502)$, Mg and $K$ content $(r=0.606)$, and $K$ and $P$ content $(r=0.636)$. However, the study showed a significant negative $(p<0.05)$ correlation between the $K$ and Na content $(\mathrm{r}=-0.126)$, and a significant $(p<0.05)$ but weak correlation between $\mathrm{P}$ and $\mathrm{Na}(\mathrm{r}=-0.052)$.

Table 8. Pearson correlation coefficients of the macromineral contents of yellow cassava roots (dataset 1 and 2).

\begin{tabular}{|c|c|c|c|c|c|c|}
\hline Variables & $\mathrm{Ca}$ & Mg & $\mathbf{N a}$ & $\mathbf{K}$ & $\mathbf{P}$ & $S$ \\
\hline \multicolumn{7}{|l|}{ Trial set 1} \\
\hline $\mathrm{Ca}$ & $1.00^{* * *}$ & & & & & \\
\hline $\mathrm{Mg}$ & $0.291^{* * *}$ & $1.00^{* * *}$ & & & & \\
\hline $\mathrm{Na}$ & 0.099 & $0.193^{* * *}$ & $1.00^{* * *}$ & & & \\
\hline K & $0.274^{* * *}$ & 0.074 & $-0.271^{* * *}$ & $1.00^{* * *}$ & & \\
\hline $\mathrm{P}$ & 0.14 * & -0.062 & -0.073 & $0.513^{* * *}$ & $1.00^{* * *}$ & \\
\hline S & $0.17^{* * *}$ & 0.024 & -0.067 & $0.397^{* * *}$ & $0.276^{* * *}$ & $1.00^{* * *}$ \\
\hline Variables & $\mathrm{Na}$ & $\mathrm{Ca}$ & $\mathrm{Mg}$ & $\mathbf{K}$ & $\mathbf{P}$ & $S$ \\
\hline \multicolumn{7}{|l|}{ Trial set 2} \\
\hline $\mathrm{Na}$ & $1.00^{* * *}$ & & & & & \\
\hline $\mathrm{Ca}$ & $0.341^{* * *}$ & $1.00^{* * *}$ & & & & \\
\hline $\mathrm{Mg}$ & $0.214^{* * *}$ & $0.605^{* * *}$ & $1.00 * * *$ & & & \\
\hline K & -0.126 & $0.502^{* * *}$ & $0.606^{* * *}$ & $1.00^{* * *}$ & & \\
\hline $\mathrm{P}$ & -0.052 & $0.343^{* * *}$ & $0.306^{* * *}$ & $0.636^{* * *}$ & $1.00^{* * *}$ & \\
\hline$S$ & 0.054 & 0.097 & $0.382^{* * *}$ & $0.23^{* * *}$ & $0.216^{* * *}$ & $1.00^{* * *}$ \\
\hline
\end{tabular}

The Pearson correlation coefficients of the micromineral contents in the yellow root cassava samples investigated are presented in Table 9. From the data set 1 trial, there was a significant positive $(p<0.001)$ correlation between the Mn and B contents $(r=0.539)$. This correlation shows the possibility of breeding yellow root cassava varieties with high $\mathrm{Mn}$ and B mineral contents. However, the results 
of the study also showed that there was a significant negative $(p<0.05)$ correlation between the Fe and $\mathrm{Ni}$ contents $(\mathrm{r}=-0.22)$, and $\mathrm{Al}$ and Ni contents $(\mathrm{r}=-0.228)$. Further, a significant $(p<0.05)$ but a weak correlation was observed between the B and Mo contents $(r=-0.161), \mathrm{Cu}$ and $\mathrm{Al}$ contents $(\mathrm{r}=-0.186)$, as well as the $\mathrm{Zn}$ and $\mathrm{Al}$ contents $(\mathrm{r}=-0.141)$.

Table 9. Pearson correlation coefficients of the micromineral contents of yellow cassava roots (dataset 1 and 2).

\begin{tabular}{|c|c|c|c|c|c|c|c|c|c|}
\hline Variables & $\mathrm{Fe}$ & Mn & B & $\mathrm{Cu}$ & Mo & Co & $\mathrm{Ni}$ & $\mathrm{Zn}$ & Al \\
\hline \multicolumn{10}{|l|}{ Trial set 1} \\
\hline $\mathrm{Fe}$ & $1.00^{* * *}$ & $0.391^{* * *}$ & $0.345^{* * *}$ & 0.056 & 0.026 & -0.029 & $-0.22^{* * *}$ & $0.178^{* *}$ & $0.425^{* * *}$ \\
\hline $\mathrm{Mn}$ & $0.392^{* * *}$ & $1.00^{* * *}$ & $0.539^{* * *}$ & $0.184^{* * *}$ & -0.053 & 0.05 & $0.16^{*}$ & $0.322 * * *$ & 0.042 \\
\hline B & $0.345^{* * *}$ & $0.539^{* * *}$ & $1.00^{* * *}$ & $0.22 * * *$ & $-0.161^{* * *}$ & -0.112 & 0.001 & $0.297^{* * *}$ & -0.084 \\
\hline $\mathrm{Cu}$ & 0.056 & $0.184^{* * *}$ & $0.22 * * *$ & $1.00^{* * *}$ & -0.071 & -0.032 & $0.316^{* * *}$ & $0.377^{* * *}$ & $-0.186^{* * *}$ \\
\hline Mo & 0.026 & -0.052 & $-0.161 *$ & -0.071 & $1.00^{* * *}$ & $0.457^{* * *}$ & -0.063 & -0.084 & $0.401^{* * *}$ \\
\hline Co & -0.029 & 0.05 & -0.112 & -0.032 & $0.457^{* * *}$ & $1.00^{* * *}$ & 0.066 & -0.036 & $0.183^{* *}$ \\
\hline $\mathrm{Ni}$ & $-0.22 * * *$ & $0.16^{*}$ & 0.001 & $0.316^{* * *}$ & -0.063 & 0.066 & $1.00^{* * *}$ & $0.189^{* * *}$ & $-0.228^{* * *}$ \\
\hline $\mathrm{Zn}$ & $0.178^{* *}$ & $0.322 * * *$ & $0.297^{* * *}$ & $0.377^{* * *}$ & -0.084 & -0.036 & $0.189^{* * *}$ & $1.00^{* * *}$ & -0.141 * \\
\hline $\mathrm{Al}$ & $0.424^{* * *}$ & 0.042 & -0.084 & $-0.186^{* * *}$ & $0.401^{* * *}$ & $0.183^{* *}$ & $-0.228^{* * *}$ & $-0.141^{*}$ & $1.00^{* * *}$ \\
\hline \multicolumn{10}{|l|}{ Trial set 2} \\
\hline $\mathrm{Fe}$ & $1.00^{* * *}$ & $0.419^{* * *}$ & $0.663^{* * *}$ & $0.341^{* * *}$ & $0.64^{* * *}$ & $0.625^{* * *}$ & $0.389^{* * *}$ & $0.229^{* * *}$ & $0.536^{* * *}$ \\
\hline Mn & $0.419^{* * *}$ & $1.00^{* * *}$ & $0.434^{* * *}$ & $0.246^{* * *}$ & $0.431^{* * *}$ & $0.446^{* * *}$ & $0.262^{* * *}$ & $0.618^{* * *}$ & $0.177^{*}$ \\
\hline B & $0.663^{* * *}$ & $0.434^{* * *}$ & $1.00^{* * *}$ & $0.32 * * *$ & $0.789 * * *$ & $0.783^{* * *}$ & $0.363^{* * *}$ & $0.249^{* * *}$ & $0.532^{* * *}$ \\
\hline $\mathrm{Cu}$ & $0.341^{* * *}$ & $0.246^{* * *}$ & $0.32 * * *$ & $1.00^{* * *}$ & $0.256^{* * *}$ & $0.266^{* * *}$ & 0.142 & $0.26^{* * *}$ & 0.183 * \\
\hline Mo & $0.64^{* * *}$ & $0.431^{* * *}$ & $0.789^{* * *}$ & $0.256^{* * *}$ & $1.00^{* * *}$ & $0.991 * * *$ & $0.27 * * *$ & $0.27^{* * *}$ & $0.603^{* * *}$ \\
\hline Co & $0.625^{* * *}$ & $0.446^{* * *}$ & $0.783^{* * *}$ & $0.266^{* * *}$ & $0.991^{* * *}$ & $1.00^{* * *}$ & $0.27^{* * *}$ & $0.283^{* * *}$ & $0.604^{* * *}$ \\
\hline $\mathrm{Ni}$ & $0.389^{* * *}$ & $0.262^{* * *}$ & $0.363^{* * *}$ & 0.142 & $0.27^{* * *}$ & $0.27^{* * *}$ & $1.00^{* * *}$ & 0.052 & 0.089 \\
\hline $\mathrm{Zn}$ & $0.229^{* * *}$ & $0.618^{* * *}$ & $0.249^{* * *}$ & $0.26^{* * *}$ & $0.27^{* * *}$ & $0.283^{* * *}$ & 0.052 & $1.00^{* * *}$ & 0.069 \\
\hline $\mathrm{Al}$ & $0.536^{* * *}$ & $0.177^{*}$ & $0.532^{* * *}$ & $0.183^{*}$ & $0.603^{* * *}$ & $0.604^{* * *}$ & 0.089 & 0.069 & $1.00^{* * *}$ \\
\hline
\end{tabular}

From the data set 2 trial, the results of the study showed a significant positive $(p<0.001)$ correlation between the Mo and Co contents $(r=0.991)$, B and Mo contents $(r=0.789), B$ and Co contents $(r=0.783)$, Fe and B contents $(r=0.663), M n$ and $Z n$ contents $(r=0.618)$, Fe and Co contents $(r=0.625), C o$ and $\mathrm{Al}$ contents $(\mathrm{r}=0.604)$, Mo and $\mathrm{Al}$ contents $(\mathrm{r}=0.603)$, Fe and $\mathrm{Al}$ contents $(\mathrm{r}=0.536)$, and $\mathrm{B}$ and $\mathrm{Al}$ contents $(\mathrm{r}=0.532)$. However, the study also showed that there was no correlation between the $\mathrm{Al}$ and $\mathrm{Ni}(\mathrm{r}=0.089), \mathrm{Al}$ and $\mathrm{Zn}(\mathrm{r}=0.069)$, and $\mathrm{Ni}$ and $\mathrm{Cu}(\mathrm{r}=0.142)$ contents.

The observed strong positive correlation shows that those minerals could be bred together to increase their concentrations in yellow cassava roots. However, those minerals with a negative and significant correlation could be difficult to improve together in the breeding program as one will increase, and the other will decrease. Curiously, both for macro- and microminerals, $\mathrm{r}$ values $>0.5$ and lower $p$-values were found for set 2 than set 1 , and the levels of minerals were higher than set 1.

\section{Conclusions}

The most abundant macromineral in cassava storage roots was found to be potassium (K), and the lowest was sodium ( $\mathrm{Na}$ ). It implies that cassava, with a high level of $\mathrm{K}$ and low $\mathrm{Na}$, might be an ideal crop to consume to reduce hypertension, although this needs further clinical evidence. The most abundant microelement was manganese (Mn), and the least abundant microelements were molybdenum (Mo) and cobalt (Co). The genotype and method had a strong influence on the concentrations of the macroand microelements in yellow-fleshed cassava roots. Most of the genotypes had higher levels of mineral density than the check varieties, which are white-fleshed, and they could be advanced to the next stage in the breeding program. Genotype 00/0028 had a high level of most of the minerals, especially Fe, $\mathrm{Zn}, \mathrm{Mn}$, and B, and could be considered to be an outstanding genotype. It could be concluded that harvesting time plays a significant role in the concentration level of some macro- and microelements in cassava roots while showing no effect on some. However, it was confirmed that yellow-fleshed roots contained more macro- and microelements than the white-fleshed roots. Method 1 (where samples were taken from all the parts and averaged) was found to be the best, but it is not cost-effective. 
Method 2 could be the best alternative because it is cost-effective but is not as accurate as method 1. The positively correlated elements could be bred together by breeders for improvements of the macro- and microelements in cassava roots. The quantitative and qualitative information on the distribution of macro- and microelements and their concentrations within the cassava tuber (proximal, middle, and distal root portions) would mostly benefit cassava breeders, food analysts, processors, and nutritionists. Yellow-fleshed cassava roots could be a good source of macro- and micro elements, and its potential as a functional food needs to be explored.

Author Contributions: Conceptualization B.M.-D., A.G.D., and E.O.A.; methodology, E.O.A., and B.M.-D.; software, E.O.A.; validation, B.M.-D., A.G.D. and E.P.; formal analysis, C.S. and E.O.A.; investigation, A.G.D. B.M.-D., E.O.A., and E.P.; resources, A.G.D. and B.M.-D.; data curation, C.S. and E.O.A.; writing-original draft preparation, E.O.A., and C.S.; writing-review and editing, B.M.-D., A.G.D. and E.P.; supervision, B.M.-D. and A.G.D.; funding acquisition, A.G.D. All authors have read and agreed to the published version of the manuscript.

Funding: This research received no external funding.

Acknowledgments: The authors acknowledged the supports from the HarvestPlus, CGIAR Research Program on Roots, Tubers, and Bananas (RTB), the staff of Food and Nutrition Sciences Laboratory and Cassava Breeding Unit, IITA, Ibadan, Nigeria.

Conflicts of Interest: The authors declare no conflict of interest.

\section{References}

1. Falah, S.A.; Saja, N.M. Essential Trace Elements and Their Vital Roles in Human Body. Indian J. Adv. Chem. Sci. 2017, 5, 127-136. [CrossRef]

2. FAO. The World Cassava Economy: Facts, Trends, and Outlooks; Food and Agriculture Organization of the United Nations and International Fund for Agricultural Development: Rome, Italy, 2000; p. 59.

3. Adugna, B. Review on Nutritional Value of Cassava for Use as a Staple Food. Sci. J. Anal. Chem. 2019, 7 , 83-91. [CrossRef]

4. Burrell, M.M. Starch: The need for improved quality or quantity-An overview. J. Exp. Bot. 2003, 54, 451-456. [CrossRef]

5. Nweke, F.I.; Spencer, D.S.; Lynam, J.K. The Cassava Transformation: Africa's Best-Kept Secret; Michigan State University Press: East Lansing, MI, USA, 2001; p. 273, ISBN 13 978-0870136023.

6. Akinpelu, A.O.; Amamgbo, L.E.F.; Olojede, A.O.; Oyekale, A.S. Health implications of cassava production and consumption. J. Agric. Soc. Res. 2011, 2, 118-125.

7. Alamu, E.O.; Ntawuruhunga, P.; Chibwe, T.; Mukuka, I.; Chiona, M. Evaluation of cassava processing and utilization at the household level in Zambia. Food Secur. 2019, 11, 141-150. [CrossRef]

8. Maziya-Dixon, B.; Alamu, E.O.; Popoola, I.O.; Yomeni, M. Nutritional and sensory properties: Snack food made from high-quality cassava flour and legume blend. Food Sci. Nutr. 2017, 5, 805-881. [CrossRef] [PubMed]

9. Hong, K.; Ma, Y.; Li, M. Solid-state fermentation of phytase from cassava dregs. Appl. Biochem. Biotechnol. 2001, 91, 777-785. [CrossRef]

10. Gomes, B.S.; Pereira, J.B., Jr.; Nunes, P.O.; Lemos, M.S.; Dantas Filho, H.A.; Dantas, K.G.F. Assessment of the Concentration of Trace Elements in Cassava Flour (Manihot esculenta Crantz) by Inductively Coupled Plasma Optical Emission Spectrometry. Rev. Virtual Quim. 2017, 9, 1699-1711. [CrossRef]

11. Golden, M.H.N. The development of concepts of malnutrition. J. Nutr. 2002, 132, 2117S-2122S. [CrossRef] [PubMed]

12. Jâkobsone, I.; Kantâne, I.; Zute, S.; Jansone, I.; Bartkeviès, V. Macro-elements and trace elements in cereal grains cultivated in Latvia. Proc. Latv. Acad. Sci. 2015, 69, 152-157. [CrossRef]

13. Maziya-Dixon, B.; Alamu, E.O.; Dixon, G.A. Variation in the evaluation of cis- and trans-carotene in yellow-fleshed cassava (Manihot esculenta Cranz) varieties as a function of the storage root portion and sampling method. LWT Food Sci. Technol. 2016, 70, 296-301. [CrossRef]

14. Wheal, M.S.; Fowles, T.O.; Palmer, L.T. A cost-effective acid digestion method using closed polypropylene tubes for inductively coupled plasma optical emission spectrometry (ICP-OES) analysis of essential plant elements. Anal. Methods 2011, 3, 2854. [CrossRef]

15. SAS. Statistical Analysis Systems (SAS) Software for Windows; SAS Institute Inc.: Cary, NC, USA, 2001. 
16. Aro, S.O.; Aletor, V.A.; Tewe, O.O.; Agbede, J.O. Nutritional potentials of cassava tuber wastes: A case study of a cassava starch processing factory in south-western Nigeria, 2010. Livest. Res. Rural. Dev. 2010, 22, 1-11.

17. Bamidele, O.P.; Fasogbon, M.B.; Oladiran, D.A.; Akande, E.O. Nutritional composition of fufu analog flour produced from Cassava root (Manihot esculenta) and Cocoyam (Colocasia esculenta) tuber. Food Sci. Nutr. 2015, 3, 597-603. [CrossRef] [PubMed]

18. Manano, J.; Ogwok, P.; Byarugaba-Bazirake, G.W. Chemical Composition of Major Cassava Varieties in Uganda, Targeted for Industrialisation. J. Field Robot. 2017, 7, 1. [CrossRef]

19. Chiwona-Karltun, L.; Nyirenda, D.; Mwansa, C.N.; Kongor, J.E.; Brimer, L.; Haggblade, S.; Afoakwa, E.O. Farmer Preference, Utilisation, and Biochemical Composition of Improved Cassava (Manihot esculenta Crantz) Varieties in Southeastern Africa. Econ. Bot. 2015, 69, 42-56. [CrossRef]

20. Otache, M.A.; Ubwa, S.T.; Agbajor, K.G. Proximate Analysis and Mineral Composition of Peels of Three Sweet Cassava Cultivars. Asian J. Phys. Chem. Sci. 2017, 3, 1-10. [CrossRef]

21. Afoakwa, E.O.; Asiedu, C.; Budu, A.S.; Chiwona-Karltun, L.; Nyirenda, D.B. Chemical composition and cyanogenic potential of traditional and high yielding CMD resistant cassava (Manihot esculenta Crantz) varieties. Int. Food Res. J. 2012, 19, 175-181.

22. Chavez, A.L.; Bedoya, J.M.; Sánchez, T.; Iglesias, C.; Ceballos, H.; Roca, W. Iron, carotene, and ascorbic acid in cassava roots and leaves. Food Nutr. Bull. 2000, 21, 410-413. [CrossRef]

23. Richardson, K.V.A. Quality Characteristics, Root Yield and Nutrient Composition of Six Cassava (Manihot Esculenta Crantz) Varieties; Gladstone Road Agricultural Centre Crops Research Report No. 18; Gladstone Road Agricultural Centre: Nassau, Bahamas, 2013.

24. Burns, E.A.; Gleadow, R.M.; Zacarias, A.M.; Cuambe, C.E.; Miller, R.E.; Cavagnaro, T.R. Variations in the Chemical Composition of Cassava (Manihot Esculenta Crantz) leaves and roots as affected by genotypic and environment variations. J. Agric. Food Chem. 2012, 60, 4946-4956. [CrossRef] [PubMed]

(C) 2020 by the authors. Licensee MDPI, Basel, Switzerland. This article is an open access article distributed under the terms and conditions of the Creative Commons Attribution (CC BY) license (http://creativecommons.org/licenses/by/4.0/). 